ESAIM: M2AN 51 (2017) 1367-1385

DOI: $10.1051 / \mathrm{m} 2 \mathrm{an} / 2016066$
ESAIM: Mathematical Modelling and Numerical Analysis

www.esaim-m2an.org

\title{
FINITE ELEMENT QUASI-INTERPOLATION AND BEST APPROXIMATION*
}

\author{
Alexandre Ern $^{1}$ And JeAn-LuC Guermond ${ }^{2}$
}

\begin{abstract}
This paper introduces a quasi-interpolation operator for scalar- and vector-valued finite element spaces constructed on affine, shape-regular meshes with some continuity across mesh interfaces. This operator gives optimal estimates of the best approximation error in any $L^{p}$-norm assuming regularity in the fractional Sobolev spaces $W^{r, p}$, where $p \in[1, \infty]$ and the smoothness index $r$ can be arbitrarily close to zero. The operator is stable in $L^{1}$, leaves the corresponding finite element space point-wise invariant, and can be modified to handle homogeneous boundary conditions. The theory is illustrated on $H^{1}$-, $\boldsymbol{H}$ (curl)- and $\boldsymbol{H}$ (div)-conforming spaces.
\end{abstract}

Mathematics Subject Classification. 65D05, 65N30, 41A65.

Received February 24, 2016. Revised September 12, 2016. Accepted October 12, 2016.

\section{INTRODUCTION}

Consider a shape-regular sequence of affine meshes $\left(\mathcal{T}_{h}\right)_{h>0}$ approximating a bounded polyhedral Lipschitz domain $D$ in $\mathbb{R}^{d}$, and a sequence of finite element spaces $\left(P\left(\mathcal{T}_{h}\right)\right)_{h>0}$ based on this mesh sequence, composed of either scalar- or vector-valued functions, and conforming in some functional space $W$ where some continuity across mesh interfaces is enforced. Estimates of the best-approximation error in $P\left(\mathcal{T}_{h}\right)$ are invariably invoked in the convergence analysis of finite element approximations. When the exact solution is smooth enough, the canonical interpolation operator in $P\left(\mathcal{T}_{h}\right)$ can be used to obtain decay estimates of the best-approximation error in terms of the mesh-size. However, in many practical situations, the exact solution only sits in a Sobolev space $W^{r, p}(D)$ (for some $p \in[1, \infty]$ ) where the smoothness index $r$ can be close to zero. In this case, an alternative quasi-interpolation operator must be invoked to estimate the best-approximation error.

The aim of this paper is the construction of a quasi-interpolation operator that is stable in $L^{1}$, leaves $P\left(\mathcal{T}_{h}\right)$ point-wise invariant, and approximates (quasi-)locally and optimally functions in $W^{r, p}(D)$, for all $p \in[1, \infty]$ and $r$ arbitrarily close to zero. Moreover, the construction can be modified to enforce homogeneous boundary conditions that are legitimate in $W$. The main examples we have in mind are $H^{1}-, \boldsymbol{H}$ (curl)-, and $\boldsymbol{H}$ (div)-conforming finite element spaces. Let us emphasize that, for vector-valued elements, the present construction is not a substitute to the notion of commuting bounded cochain projection introduced in the framework of Finite Element

Keywords and phrases. Quasi-interpolation, finite elements, best approximation.

* This material is based upon work supported in part by the National Science Foundation grant DMS-1217262, by the Air Force Office of Scientific Research, USAF, under grant/contract number FA9550-15-1-0257 and by the Army Research Office under grant number W911NF-15-1-0517, Draft version, 19th July 2017

1 Université Paris-Est, CERMICS (ENPC), 77455 Marne-la-Vallée cedex 2, France.

2 Department of Mathematics, Texas A\&M University 3368 TAMU, College Station, TX 77843, USA. guermond@math.tamu.edu 
Exterior Calculus, but instead a vital complement to it. Indeed, as shown in (Arnold et al. [2], Thm. 5.6), the approximation error for the commuting projection is bounded by the best-approximation error in $P\left(\mathcal{T}_{h}\right)$, up to a uniform constant. Therefore, the present quasi-interpolation operator shows that commuting bounded cochain projections converge optimally for low-regularity solutions. Another important consequence of the present construction in the case of vector-valued elements is that the decay rates of the best-approximation error only involve the solution seminorm in $W^{r, p}(D)$ without the need to invoke a bound on the curl or the divergence of the solution.

Let us put our results in perspective with the literature. The construction of quasi-interpolation operators with the above properties for $H^{1}$-conforming finite elements is a well-studied field. In two space dimensions on triangular meshes, we mention the early work of Clément [9]; see also Bernardi and Girault [3] where the construction is modified so that the resulting operator leaves the finite element space point-wise invariant. In a landmark paper by Scott and Zhang [23], an alternative construction is proposed in any space dimension for $H^{1}$ conforming finite elements with and without homogeneous boundary conditions; see [23], page 491, and Girault and Lions [15] for a modification leading to $L^{1}$-stability. The so-called Scott-Zhang interpolation operator leads to optimal decay estimates of the approximation error for functions in $H^{1}(D)$. The extension of this result to functions in $W^{r, p}(D)$ with $r \in\left(\frac{1}{p}, 1\right)$ has been studied only recently by Ciarlet [7] using the original Scott-Zhang operator. To the authors' best knowledge, in contrast to $H^{1}$-conforming elements, quasi-interpolation results for $\boldsymbol{H}$ (curl)- and $\boldsymbol{H}$ (div)-conforming elements are missing in the literature.

The main ingredient for the Clément and Scott-Zhang construction is a regularization of functions based on macroelements consisting of patches of elements. We adopt here a somewhat different route. The key idea is a two-step construction, namely a projection onto the (fully discontinuous) broken finite element space followed by an averaging operator that stitches the result in the spirit of (Oswald [20], Eqs. (25), (26)). This way, patches of mesh cells are only involved when handling discrete objects, thereby avoiding the delicate question of reference patches frequently used in the literature. Averaging operators are often invoked in the literature in various contexts; we mention, in particular, a posteriori error estimates $[1,18,14]$, preconditioners [22], stabilization techniques [5], and discontinuous Galerkin methods with improved stability properties [10,6]. The novel feature here is the analysis of averaging operators in the vector-valued case and the application to quasi-interpolation. We emphasize that we devise a unifying framework for the analysis that works irrespective of the nature of the degrees of freedom, i.e. whether they are nodal values, integrals over edges, faces, or cells. Essentially all the finite elements that are traditionally used to build $H^{1}-, \boldsymbol{H}$ (curl)-, and $\boldsymbol{H}$ (div)-conforming finite element spaces match the few assumptions of the unified analysis.

The paper is organized as follows. In Section 2, we introduce the notation and construct a sequence of abstract finite element spaces conforming in some functional space $W$. Key assumptions are identified and listed. A local interpolation operator stable in $L^{1}$ is constructed in Section 3. An abstract averaging operator acting only on discrete functions is introduced and analyzed in Section 4. The final quasi-interpolation operator is constructed in Section 5 without enforcing any boundary condition. The question of enforcing boundary conditions is addressed in Section 6. Finally, Section 7 contains two technical results on fractional Sobolev spaces that are of independent interest: a Poincaré inequality and a trace inequality.

\section{Finite Elements}

In this section we introduce some notation and construct a sequence of abstract finite element spaces, conforming in some functional space $W$. In the entire paper the space dimension is denoted by $d$ and the domain $D$ is a bounded Lipschitz polyhedron in $\mathbb{R}^{d}$.

\subsection{Meshes}

Let $\left(\mathcal{T}_{h}\right)_{h>0}$ be a mesh sequence that we assume to be affine and shape-regular in the sense of Ciarlet. We also assume for the sake of simplicity that the meshes cover $D$ exactly, that they are composed of convex cells, and that they are matching, i.e. for all cells $K, K^{\prime} \in \mathcal{T}_{h}$ such that $K \neq K^{\prime}$ and $K \cap K^{\prime} \neq \emptyset$, the set $K \cap K^{\prime}$ 
is a common vertex, edge, or face of both $K$ and $K^{\prime}$ (with obvious extensions in higher space dimensions). By convention, given a mesh $\mathcal{T}_{h}$, the elements $K \in \mathcal{T}_{h}$ are closed sets in $\mathbb{R}^{d}$.

We assume that there is a reference element $\widehat{K}$ such that for any mesh $\mathcal{T}_{h}$ and any cell $K \in \mathcal{T}_{h}$, there is an affine bijective map between $\widehat{K}$ and $K$, which we henceforth denote $\boldsymbol{T}_{K}: \widehat{K} \rightarrow K$. Since $\boldsymbol{T}_{K}$ is affine and bijective, there is an invertible matrix $\mathbb{J}_{K} \in \mathbb{R}^{d \times d}$ such that

$$
\boldsymbol{T}_{K}(\widehat{\boldsymbol{x}})-\boldsymbol{T}_{K}(\widehat{\boldsymbol{y}})=\mathbb{J}_{K}(\widehat{\boldsymbol{x}}-\widehat{\boldsymbol{y}}), \quad \forall \widehat{\boldsymbol{x}}, \widehat{\boldsymbol{y}} \in \widehat{K} .
$$

In what follows, we denote points in $\mathbb{R}^{d}, \mathbb{R}^{d}$-valued functions and $\mathbb{R}^{d}$-valued maps in boldface type, and we denote the Euclidean norm in $\mathbb{R}^{d}$ by $\|\cdot\|_{\ell^{2}\left(\mathbb{R}^{d}\right)}$, or $\|\cdot\|_{\ell^{2}}$ when the context is unambiguous. We abuse the notation by using the same symbol for the induced matrix norm. Owing to the shape-regularity assumption of the mesh sequence, there are uniform constants $c^{\sharp}, c^{b}$ such that

$$
\left|\operatorname{det}\left(\mathbb{J}_{K}\right)\right|=|K||\widehat{K}|^{-1}, \quad\left\|\mathbb{J}_{K}\right\|_{\ell^{2}} \leq c^{\sharp} h_{K}, \quad\left\|\mathbb{J}_{K}^{-1}\right\|_{\ell^{2}} \leq c^{b} h_{K}^{-1},
$$

where $h_{K}$ is the diameter of $K$. Recall that $c^{\sharp}=\frac{1}{\rho_{\widehat{K}}}$ and $c^{b}=\frac{h_{K}}{\rho_{K}} h_{\widehat{K}}$ for meshes composed of simplices, where $\rho_{K}$ is the diameter of the largest ball that can be inscribed in $K, h_{\widehat{K}}$ is the diameter of $\widehat{K}$, and $\rho_{\widehat{K}}$ is the diameter of the largest ball that can be inscribed in $\widehat{K}$.

\subsection{Finite element generation}

We are going to consider various approximation spaces based on the mesh sequence $\left(\mathcal{T}_{h}\right)_{h>0}$. Again for the sake of simplicity, we assume that each approximation space is constructed from a fixed reference finite element $(\widehat{K}, \widehat{P}, \widehat{\Sigma})$. The reference degrees of freedom are denoted $\left\{\widehat{\sigma}_{1}, \ldots, \widehat{\sigma}_{n_{\mathrm{sh}}}\right\}$ and the associated reference shape functions are denoted $\left\{\widehat{\theta}_{1}, \ldots, \widehat{\theta}_{n_{\text {sh }}}\right\}$; by definition $\widehat{\sigma}_{i}\left(\widehat{\theta}_{j}\right)=\delta_{i j}, \forall i, j \in\left\{1: n_{\text {sh }}\right\}$. We denote $\mathcal{N}:=\left\{1: n_{\text {sh }}\right\}$ to alleviate the notation. The shape functions are $\mathbb{R}^{q}$-valued for some integer $q \geq 1$. We henceforth assume that $\widehat{P} \subset W^{1, \infty}\left(\widehat{K} ; \mathbb{R}^{q}\right)$ (recall that $\widehat{P}$ is typically a space of polynomial functions, but we do not require this assumption here).

We assume that there exists a Banach space $V(\widehat{K}) \subset L^{1}\left(\widehat{K} ; \mathbb{R}^{q}\right)$ such that the linear forms $\left\{\widehat{\sigma}_{1}, \ldots, \widehat{\sigma}_{n_{\mathrm{sh}}}\right\}$ can be extended to $\mathcal{L}(V(\widehat{K}) ; \mathbb{R})$, i.e. $V(\widehat{K})$ is the domain of the degrees of freedom (see [13], p. 39). Then, we define $\mathcal{I}_{\widehat{K}}: V(\widehat{K}) \rightarrow \widehat{P}$, the interpolation operator associated with the reference finite element $(\widehat{K}, \widehat{P}, \widehat{\Sigma})$, by

$$
\mathcal{I}_{\widehat{K}}(\widehat{v})(\widehat{\boldsymbol{x}})=\sum_{i \in \mathcal{N}} \widehat{\sigma}_{i}(v) \widehat{\theta}_{i}(\widehat{\boldsymbol{x}}), \quad \forall \widehat{\boldsymbol{x}} \in \widehat{K}, \quad \forall \widehat{v} \in V(\widehat{K}) .
$$

By construction, $\mathcal{I}_{\widehat{K}} \in \mathcal{L}(V(\widehat{K}) ; \widehat{P})$, and $\widehat{P}$ is point-wise invariant under $\mathcal{I}_{\widehat{K}}$.

We now address the question of constructing finite elements for any mesh cell $K \in \mathcal{T}_{h}$. We assume that there exists a Banach space $V(K) \subset L^{1}\left(K ; \mathbb{R}^{q}\right)$ and a bounded, bijective, linear map between $V(K)$ and $V(\widehat{K})$ :

$$
\psi_{K}: V(K) \ni v \longmapsto \psi_{K}(v) \in V(\widehat{K}) .
$$

We then set

$$
\begin{aligned}
& P_{K}:=\left\{p=\psi_{K}^{-1}(\widehat{p}) \mid \widehat{p} \in \widehat{P}\right\}, \\
& \Sigma_{K}:=\left\{\sigma_{K, i}\right\}_{i \in \mathcal{N}} \text { s.t. } \sigma_{K, i}=\widehat{\sigma}_{i} \circ \psi_{K} .
\end{aligned}
$$


Proposition 2.1 (Finite element). The triple $\left(K, P_{K}, \Sigma_{K}\right)$ is a finite element.

Proof. Note first that $\operatorname{dim}\left(P_{K}\right)=\operatorname{dim}(\widehat{P})=n_{\text {sh }}$ since $\psi_{K}$ is bijective. Moreover, a function $p \in P_{K}$ such that $\sigma_{K, i}(p)=0$ for all $i \in \mathcal{N}$ is such that $\psi_{K}(p)=0$ by the unisolvence property of the reference finite element; hence, $p=0$. Finally, the linear forms $\sigma_{K, i}$ are in $\mathcal{L}(V(K) ; \mathbb{R})$ since $\left|\sigma_{K, i}(v)\right| \leq\left\|\widehat{\sigma}_{i}\right\|_{\mathcal{L}(V(\widehat{K}) ; \mathbb{R})}\left\|\psi_{K}\right\|_{\mathcal{L}(V(K) ; V(\widehat{K}))}\|v\|_{V(K)}$, for all $v \in V(K)$.

The above definitions lead us to consider the canonical interpolation operator associated with the finite element $\left(K, P_{K}, \Sigma_{K}\right)$ :

$$
\mathcal{I}_{K}(v)(\boldsymbol{x})=\sum_{i \in \mathcal{N}} \sigma_{K, i}(v) \theta_{K, i}(\boldsymbol{x}), \quad \forall \boldsymbol{x} \in K, \quad \forall v \in V(K),
$$

where we have set $\theta_{K, i}:=\psi_{K}^{-1}\left(\widehat{\theta}_{i}\right)$. Note that $\mathcal{I}_{K} \in \mathcal{L}\left(V(K) ; P_{K}\right)$ and that $P_{K}$ is point-wise invariant under $\mathcal{I}_{K}$.

Since the mesh is affine, we assume that $\psi_{K}$ has a simple structure; more precisely, we assume that there is a $q \times q$ invertible matrix $\mathbb{A}_{K}$ such that

$$
\psi_{K}(v)=\mathbb{A}_{K}\left(v \circ \boldsymbol{T}_{K}\right),
$$

which implies that $\psi_{K}$ can be extended as a map from $L^{1}\left(K ; \mathbb{R}^{q}\right)$ to $L^{1}\left(\widehat{K} ; \mathbb{R}^{q}\right)$. The following classical result shows that $\psi_{K}$ maps $W^{l, p}\left(K ; \mathbb{R}^{q}\right)$ to $W^{l, p}\left(\widehat{K} ; \mathbb{R}^{q}\right)$ for all $l \in \mathbb{N}$ and all $p \in[1, \infty]$. In particular, this implies that $P_{K} \subset W^{1, \infty}\left(K ; \mathbb{R}^{q}\right)$. We use the notation $\left|\psi_{K}\right|_{\mathcal{L}\left(W^{l, p}\left(K ; \mathbb{R}^{q}\right) ; W^{l, p}\left(\widehat{K} ; \mathbb{R}^{q}\right)\right)}:=\sup _{v \in W^{l, p}\left(K ; \mathbb{R}^{q}\right)} \frac{\left|\psi_{K}(v)\right|_{W^{l, p}\left(\widehat{K} ; \mathbb{R}^{q}\right)}}{|v|_{W^{l, p}(K ; \mathbb{R} q)}}$ and similar notation for $\left|\psi_{K}^{-1}\right|_{\mathcal{L}\left(W^{l, p}\left(\widehat{K} ; \mathbb{R}^{q}\right) ; W^{l, p}\left(K ; \mathbb{R}^{q}\right)\right)}$, where it henceforth implicitly understood the denominator is not zero.

Lemma 2.2 (Bound in Sobolev norms). Let $l \in \mathbb{N}$. There is a uniform constant $c$ depending on the shaperegularity of the mesh sequence $\left(\mathcal{T}_{h}\right)_{h>0}$ and on $l$ such that the following holds:

$$
\begin{aligned}
\left|\psi_{K}\right|_{\mathcal{L}\left(W^{l, p}\left(K ; \mathbb{R}^{q}\right) ; W^{l, p}\left(\widehat{K} ; \mathbb{R}^{q}\right)\right)} & \leq c\left\|\mathbb{A}_{K}\right\|_{\ell^{2}}\left\|\mathbb{J}_{K}\right\|_{\ell^{2}}^{l}\left|\operatorname{det}\left(\mathbb{J}_{K}\right)\right|^{-\frac{1}{p}}, \\
\left|\psi_{K}^{-1}\right|_{\mathcal{L}\left(W^{l, p}\left(\widehat{K} ; \mathbb{R}^{q}\right) ; W^{l, p}\left(K ; \mathbb{R}^{q}\right)\right)} & \leq c\left\|\mathbb{A}_{K}^{-1}\right\|_{\ell^{2}}\left\|\mathbb{J}_{K}^{-1}\right\|_{\ell^{2}}^{l}\left|\operatorname{det}\left(\mathbb{J}_{K}\right)\right|^{\frac{1}{p}},
\end{aligned}
$$

for all $K \in \mathcal{T}_{h}$ and all $p \in[1, \infty]$ (with $z^{ \pm \frac{1}{p}}=1, \forall z>0$ if $p=\infty$ ).

Proof. For any multilinear map $A \in \mathcal{M}_{l}\left(\mathbb{R}^{d}, \ldots, \mathbb{R}^{d} ; \mathbb{R}^{q}\right)$, let us set

$$
\|A\|_{\mathcal{M}_{l}\left(\mathbb{R}^{d}, \ldots, \mathbb{R}^{d} ; \mathbb{R}^{q}\right)}:=\sup _{\left(\boldsymbol{y}_{1}, \ldots, \boldsymbol{y}_{l}\right) \in \mathbb{R}^{d} \times \ldots \times \mathbb{R}^{d}} \frac{\left\|A\left(\boldsymbol{y}_{1}, \ldots, \boldsymbol{y}_{l}\right)\right\|_{\ell^{2}}}{\left\|\boldsymbol{y}_{1}\right\|_{\ell^{2}} \ldots\left\|\boldsymbol{y}_{l}\right\|_{\ell^{2}}}
$$

Then, denoting by $D^{l} \psi_{K}(v)$ the $l$-order Frechet derivative of $\psi_{K}$ at $v$, the assumption (2.7) implies that $\left\|D^{l} \psi_{K}(v)\right\|_{\mathcal{M}_{l}\left(\mathbb{R}^{d}, \ldots, \mathbb{R}^{d} ; \mathbb{R}^{q}\right)} \leq\left\|\mathbb{A}_{K}\right\|_{\ell^{2}}\left\|D^{l}\left(v \circ \boldsymbol{T}_{K}\right)\right\|_{\mathcal{M}_{l}\left(\mathbb{R}^{d}, \ldots, \mathbb{R}^{d} ; \mathbb{R}^{q}\right)}$ for all $l \in \mathbb{N}$. Then, standard results about the transformation of seminorms in the Sobolev space $W^{l, p}$ using the pullback by $\boldsymbol{T}_{K}$ lead to (2.8), see e.g. (Ciarlet [8], Thm. 3.1.2 or Ern and Guermond [13], Lem. 1.101).

Corollary 2.3 (Bound on $\mathbb{A}_{K}$ ). Assume that there is a uniform constant $c$ so that

$$
\left\|\mathbb{A}_{K}\right\|_{\ell^{2}}\left\|\mathbb{A}_{K}^{-1}\right\|_{\ell^{2}} \leq c\left\|\mathbb{J}_{K}\right\|_{\ell^{2}}\left\|\mathbb{J}_{K}^{-1}\right\|_{\ell^{2}} .
$$

Then the following holds for all $s, m \in \mathbb{N}$, all $K \in \mathcal{T}_{h}$ and all $p \in[1, \infty]$ :

$$
\left|\psi_{K}^{-1}\right|_{\mathcal{L}\left(W^{m, p}(\widehat{K}) ; W^{m, p}(K)\right)}\left|\psi_{K}\right|_{\mathcal{L}\left(W^{s, p}(K) ; W^{s, p}(\widehat{K})\right)} \leq c h_{K}^{s-m} .
$$

Proof. Combine (2.8) with (2.2) and (2.9). 
Lemma 2.4 (Norm equivalence). There exists a uniform constant $c$ such that

$$
\begin{gathered}
\|v\|_{L^{\infty}\left(K ; \mathbb{R}^{q}\right)} \leq c\left\|\mathbb{A}_{K}^{-1}\right\|_{\ell^{2}} \sum_{i \in \mathcal{N}}\left|\sigma_{K, i}(v)\right|, \\
\sum_{i \in \mathcal{N}}\left|\sigma_{K, i}(v)\right| \leq c\left\|\mathbb{A}_{K}\right\|_{\ell^{2}}\|v\|_{L^{\infty}\left(K ; \mathbb{R}^{q}\right)},
\end{gathered}
$$

for all $v \in P_{K}$ and all $K \in \mathcal{T}_{h}$.

Proof. We prove (2.11); the proof for the other bound is similar. We observe that

$$
\begin{aligned}
\|v\|_{L^{\infty}\left(K ; \mathbb{R}^{q}\right)} & =\left\|\psi_{K}^{-1}\left(\psi_{K}(v)\right)\right\|_{L^{\infty}\left(K ; \mathbb{R}^{q}\right)} \leq\left\|\mathbb{A}_{K}^{-1}\right\|_{\ell^{2}}\left\|\psi_{K}(v)\right\|_{L^{\infty}\left(\widehat{K} ; \mathbb{R}^{q}\right)} \\
& \leq c\left\|\mathbb{A}_{K}^{-1}\right\|_{\ell^{2}} \sum_{i \in \mathcal{N}}\left|\widehat{\sigma}_{i}\left(\psi_{K}(v)\right)\right|=c\left\|\mathbb{A}_{K}^{-1}\right\|_{\ell^{2}} \sum_{i \in \mathcal{N}}\left|\sigma_{K, i}(v)\right|,
\end{aligned}
$$

using the definition of $\psi_{K}$, norm equivalence in $\widehat{P}$, and (2.5b).

\subsection{Abstract finite element spaces}

Let $\left\{\left(K, P_{K}, \Sigma_{K}\right)\right\}_{K \in \mathcal{T}_{h}}$ be a $\mathcal{T}_{h}$-based family of finite elements constructed as in Proposition 2.1. We consider the broken Sobolev spaces $W^{1, p}\left(\mathcal{T}_{h} ; \mathbb{R}^{q}\right):=\left\{v \in L^{p}\left(D ; \mathbb{R}^{q}\right) \mid v_{\mid K} \in W^{1, p}\left(K ; \mathbb{R}^{q}\right), \forall K \in \mathcal{T}_{h}\right\}, p \in[1, \infty]$, and introduce the broken finite element space

$$
P^{\mathrm{b}}\left(\mathcal{T}_{h}\right)=\left\{v_{h} \in L^{1}\left(D ; \mathbb{R}^{q}\right) \mid v_{h \mid K} \in P_{K}, \forall K \in \mathcal{T}_{h}\right\} .
$$

Since $P_{K} \subset W^{1, \infty}\left(K ; \mathbb{R}^{q}\right)$, we infer that $P^{\mathrm{b}}\left(\mathcal{T}_{h}\right) \subset W^{1, \infty}\left(\mathcal{T}_{h} ; \mathbb{R}^{q}\right)$.

Our aim is to define a subspace of $P^{\mathrm{b}}\left(\mathcal{T}_{h}\right)$ by means of some zero-jump condition across the interfaces separating the elements. We say that a subset $F \subset \bar{D}$ is an interface if it has positive $(d-1)$-dimensional measure and if there are distinct mesh cells $K_{l}, K_{r} \in \mathcal{T}_{h}$ such that $F=\partial K_{l} \cap \partial K_{r}$. The numbering of the two mesh cells is arbitrary, but kept fixed once and for all, and we let $\boldsymbol{n}_{F}$ be the unit normal vector to $F$ pointing from $K_{l}$ to $K_{r}$. This defines a global orientation of the interfaces. We denote by $\boldsymbol{n}_{K_{l}}$ and $\boldsymbol{n}_{K_{r}}$ the outward unit normal of $K_{l}$ and $K_{r}$, i.e. $\boldsymbol{n}_{F}=\boldsymbol{n}_{K_{l}}=-\boldsymbol{n}_{K_{r}}$. We say that a subset $F \subset \bar{D}$ is a boundary face if it has positive $(d-1)$-dimensional measure and if there is a mesh cell $K \in \mathcal{T}_{h}$ such that $F=\partial K \cap \partial D$, and we let $\boldsymbol{n}_{F}$ be the unit normal vector to $F$ pointing outward $D$. The interfaces are collected in the set $\mathcal{F}_{h}^{\circ}$, the boundary faces in the set $\mathcal{F}_{h}^{\partial}$, and we let $\mathcal{F}_{h}=\mathcal{F}_{h}^{\circ} \cup \mathcal{F}_{h}^{\partial}$. We now define a notion of jump across the interfaces. Recalling that functions in $W^{1,1}\left(\mathcal{T}_{h} ; \mathbb{R}^{q}\right)$ have traces in $L^{1}\left(\partial K ; \mathbb{R}^{q}\right)$ for all $K \in \mathcal{T}_{h}$, let $F \in \mathcal{F}_{h}^{\circ}$ be a mesh interface, and let $K_{l}, K_{r}$ be the two cells such that $F=\partial K_{l} \cap \partial K_{r}$; the jump of $v \in W^{1,1}\left(\mathcal{T}_{h} ; \mathbb{R}^{q}\right)$ across $F$ is defined to be

$$
\llbracket v \rrbracket_{F}(\boldsymbol{x})=v_{\mid K_{l}}(\boldsymbol{x})-v_{\mid K_{r}}(\boldsymbol{x}) \quad \text { a.e. } \boldsymbol{x} \in F .
$$

In what follows, we need to consider the jump of only some components of $v$ across $F$; we formalize this by introducing bounded linear operators $\gamma_{K, F}: W^{1,1}\left(K ; \mathbb{R}^{q}\right) \rightarrow L^{1}\left(F ; \mathbb{R}^{t}\right)$, for all $K \in \mathcal{T}_{h}$, all face $F \in \mathcal{F}_{h}$ that is a subset of $\partial K$, and some $t \geq 1$, as follows:

$$
\llbracket v \rrbracket_{F}^{\gamma}(\boldsymbol{x})=\gamma_{K_{l}, F}\left(v_{\mid K_{l}}\right)(\boldsymbol{x})-\gamma_{K_{r}, F}\left(v_{\mid K_{r}}\right)(\boldsymbol{x}) \quad \text { a.e. } \boldsymbol{x} \in F .
$$

We assume that $\left|\llbracket v \rrbracket_{F}^{\gamma}(\boldsymbol{x})\right| \leq\left|\llbracket v \rrbracket_{F}(\boldsymbol{x})\right|$, a.e. $\boldsymbol{x} \in F$, for all $v \in W^{1,1}\left(\mathcal{T}_{h} ; \mathbb{R}^{q}\right)$. Since functions in $W^{1,1}\left(D ; \mathbb{R}^{q}\right)$ have zero jump across interfaces (see, e.g. [11], Lem. 1.23), we infer that

$$
v \in W^{1,1}\left(D ; \mathbb{R}^{q}\right) \Longrightarrow \llbracket v \rrbracket_{F}^{\gamma}=0, \forall F \in \mathcal{F}_{h}^{\circ} .
$$

With this setting, we introduce the

$$
P\left(\mathcal{T}_{h}\right)=\left\{v_{h} \in P^{\mathrm{b}}\left(\mathcal{T}_{h}\right) \mid \llbracket v_{h} \rrbracket_{F}^{\gamma}=0, \forall F \in \mathcal{F}_{h}^{\circ}\right\} .
$$




\subsection{Finite element examples}

The present theory is quite general and covers a large class of scalar- and vector-valued finite elements. For instance, it covers finite elements of Lagrange, Nédélec, and Raviart-Thomas type. To remain general, we denote the three reference elements corresponding to these three classes as follows: $\left(\widehat{K}, \widehat{P}^{\mathrm{g}}, \widehat{\Sigma}^{\mathrm{g}}\right),\left(\widehat{K}, \widehat{\boldsymbol{P}}^{\mathrm{c}}, \widehat{\Sigma}^{\mathrm{c}}\right)$ and $\left(\widehat{K}, \widehat{\boldsymbol{P}}^{\mathrm{d}}, \widehat{\Sigma}^{\mathrm{d}}\right)$. We think of $\left(\widehat{K}, \widehat{P}^{\mathrm{g}}, \widehat{\Sigma}^{\mathrm{g}}\right)$ as a scalar-valued finite element $(q=1)$ that has some degrees of freedom which require point evaluation, for instance $\left(\widehat{K}, \widehat{P}^{\mathrm{g}}, \widehat{\Sigma}^{\mathrm{g}}\right)$ could be a Lagrange element. We assume that the finite element $\left(\widehat{K}, \widehat{\boldsymbol{P}}^{\mathrm{c}}, \widehat{\Sigma}^{\mathrm{c}}\right)$ is vector-valued $(q=d)$ and some of its degrees of freedom require to evaluate integrals over edges. Typically, $\left(\widehat{K}, \widehat{\boldsymbol{P}}^{\mathrm{c}}, \widehat{\Sigma}^{\mathrm{c}}\right)$ is a Nédélec-type or edge element. Likewise, the finite element $\left(\widehat{K}, \widehat{\boldsymbol{P}}^{\mathrm{d}}, \widehat{\Sigma}^{\mathrm{d}}\right)$ is assumed to be vector-valued $(q=d)$ and some of its degrees of freedom are assumed to require evaluation of integrals over faces. Typically, $\left(\widehat{K}, \widehat{\boldsymbol{P}}^{\mathrm{d}}, \widehat{\Sigma}^{\mathrm{d}}\right)$ is a Raviart-Thomas-type element. It is not necessary to know the exact nature of the element that we are handling at the moment. We denote by $V^{\mathrm{g}}(\widehat{K}), \boldsymbol{V}^{\mathrm{c}}(\widehat{K}), \boldsymbol{V}^{\mathrm{d}}(\widehat{K})$ admissible domains of the degrees of freedom in the three cases. Let $p \in[1, \infty]$. The above assumptions imply that we can choose $V^{\mathrm{g}}(\widehat{K})=W^{s, p}(\widehat{K})$ with $s>\frac{d}{p}, \boldsymbol{V}^{\mathrm{c}}(\widehat{K})=\boldsymbol{W}^{s, p}(\widehat{K})$ with $s>\frac{d-1}{p}$, and $\boldsymbol{V}^{\mathrm{d}}(\widehat{K})=\boldsymbol{W}^{s, p}(\widehat{K})$ with $s>\frac{1}{p}$. Actually, when $p=1$ we can choose $V^{\mathrm{g}}(\widehat{K})=W^{d, 1}(\widehat{K})$ (since $W^{d, 1}(\widehat{K}) \hookrightarrow \mathcal{C}^{0}(\widehat{K})$, see e.g. Ponce and Van Schaftingen [21]), $\boldsymbol{V}^{\mathrm{d}}(\widehat{K})=\boldsymbol{W}^{1,1}(\widehat{K})$ (since functions in $W^{1,1}(\widehat{K})$ have a trace in $L^{1}(\partial \widehat{K})$ ), and $\boldsymbol{V}^{\mathrm{c}}(\widehat{K})=\boldsymbol{W}^{d-1,1}(\widehat{K})$ (since functions in $W^{d-1,1}(\widehat{K})$ have traces in $L^{1}$ on the one-dimensional edges of $\widehat{K}$ ).

Let $\mathcal{T}_{h}$ be a mesh in the sequence $\left(\mathcal{T}_{h}\right)_{h>0}$ and let $K$ be a cell in $\mathcal{T}_{h}$. We denote by $\psi_{K}^{\mathrm{g}}, \boldsymbol{\psi}_{K}^{\mathrm{c}}, \boldsymbol{\psi}_{K}^{\mathrm{d}}$ the linear maps that are classically used to generate the above finite elements, i.e. $\psi_{K}^{\mathrm{g}}$ is the pullback by $\boldsymbol{T}_{K}$, and $\psi_{K}^{\mathrm{c}}$ and $\psi_{K}^{\mathrm{d}}$ are the contravariant and covariant Piola transformations, respectively. All of these maps fit the general form (2.7), i.e.

$$
\begin{array}{ll}
\mathbb{A}_{K}^{\mathrm{g}}=1, & \psi_{K}^{\mathrm{g}}(v)=v \circ \boldsymbol{T}_{K}, \\
\mathbb{A}_{K}^{\mathrm{c}}=\mathbb{J}_{K}^{\mathrm{T}}, & \boldsymbol{\psi}_{K}^{\mathrm{c}}(\boldsymbol{v})=\mathbb{J}_{K}^{\mathrm{T}}\left(\boldsymbol{v} \circ \boldsymbol{T}_{K}\right), \\
\mathbb{A}_{K}^{\mathrm{d}}=\operatorname{det}\left(\mathbb{J}_{K}\right) \mathbb{J}_{K}^{-1}, & \boldsymbol{\psi}_{K}^{\mathrm{d}}(\boldsymbol{v})=\operatorname{det}\left(\mathbb{J}_{K}\right) \mathbb{J}_{K}^{-1}\left(\boldsymbol{v} \circ \boldsymbol{T}_{K}\right) .
\end{array}
$$

Note that $c=1$ in (2.9) for the above examples.

The corresponding broken finite element spaces are:

$$
\begin{aligned}
& P^{\mathrm{g}, \mathrm{b}}\left(\mathcal{T}_{h}\right)=\left\{v_{h} \in L^{1}(D) \mid \psi_{K}^{\mathrm{g}}\left(v_{h \mid K}\right) \in \widehat{P}^{\mathrm{g}}, \forall K \in \mathcal{T}_{h}\right\}, \\
& \boldsymbol{P}^{\mathrm{c}, \mathrm{b}}\left(\mathcal{T}_{h}\right)=\left\{\boldsymbol{v}_{h} \in \boldsymbol{L}^{1}(D) \mid \boldsymbol{\psi}_{K}^{\mathrm{c}}\left(\boldsymbol{v}_{h \mid K}\right) \in \widehat{\boldsymbol{P}}^{\mathrm{c}}, \forall K \in \mathcal{T}_{h}\right\}, \\
& \boldsymbol{P}^{\mathrm{d}, \mathrm{b}}\left(\mathcal{T}_{h}\right)=\left\{\boldsymbol{v}_{h} \in \boldsymbol{L}^{1}(D) \mid \boldsymbol{\psi}_{K}^{\mathrm{d}}\left(\boldsymbol{v}_{h \mid K}\right) \in \widehat{\boldsymbol{P}}^{\mathrm{d}}, \forall K \in \mathcal{T}_{h}\right\} .
\end{aligned}
$$

This leads us to consider the following $\gamma$-traces:

$$
\begin{array}{rlrl}
\gamma_{K, F}^{\mathrm{g}}\left(v_{\mid K}\right)(\boldsymbol{x}) & =v_{\mid K}(\boldsymbol{x}), & & \forall \boldsymbol{x} \in F, \\
\gamma_{K, F}^{\mathrm{c}}\left(\boldsymbol{v}_{\mid K}\right)(\boldsymbol{x})=\boldsymbol{v}_{\mid K}(\boldsymbol{x}) \times \boldsymbol{n}_{F}, & & \forall \boldsymbol{x} \in F, \\
\gamma_{K, F}^{\mathrm{d}}\left(\boldsymbol{v}_{\mid K}\right)(\boldsymbol{x})=\boldsymbol{v}_{\mid K}(\boldsymbol{x}) \cdot \boldsymbol{n}_{F}, & & \forall \boldsymbol{x} \in F,
\end{array}
$$

and the following conforming finite element spaces:

$$
\begin{aligned}
& P^{\mathrm{g}}\left(\mathcal{T}_{h}\right)=\left\{v_{h} \in P^{\mathrm{g}, \mathrm{b}}\left(\mathcal{T}_{h}\right) \mid \llbracket v_{h} \rrbracket_{F}^{\mathrm{g}}=0, \forall F \in \mathcal{F}_{h}^{\circ}\right\}, \\
& \boldsymbol{P}^{\mathrm{c}}\left(\mathcal{T}_{h}\right)=\left\{\boldsymbol{v}_{h} \in \boldsymbol{P}^{\mathrm{c}, \mathrm{b}}\left(\mathcal{T}_{h}\right) \mid \llbracket \boldsymbol{v}_{h} \rrbracket_{F}^{\mathrm{c}}=0, \forall F \in \mathcal{F}_{h}^{\circ}\right\}, \\
& \boldsymbol{P}^{\mathrm{d}}\left(\mathcal{T}_{h}\right)=\left\{\boldsymbol{v}_{h} \in \boldsymbol{P}^{\mathrm{d}, \mathrm{b}}\left(\mathcal{T}_{h}\right) \mid \llbracket \boldsymbol{v}_{h} \rrbracket_{F}^{\mathrm{d}}=0, \forall F \in \mathcal{F}_{h}^{\circ}\right\},
\end{aligned}
$$

where we slightly simplified the notation by using $\llbracket v_{h} \rrbracket_{F}^{\mathrm{g}}$ instead of $\llbracket v_{h} \rrbracket_{F}^{\gamma^{\mathrm{g}}}$, etc. Upon introducing the spaces $V^{\mathrm{g}}:=\left\{v \in L^{1}(D) \mid \nabla v \in \boldsymbol{L}^{1}(D)\right\}, \boldsymbol{V}^{\mathrm{c}}:=\left\{\boldsymbol{v} \in \boldsymbol{L}^{1}(D) \mid \nabla \times \boldsymbol{v} \in \boldsymbol{L}^{1}(D)\right\}, \boldsymbol{V}^{\mathrm{d}}:=\left\{\boldsymbol{v} \in \boldsymbol{L}^{1}(D) \mid \nabla \cdot v \in L^{1}(D)\right\}$, 
we have $P^{\mathrm{g}}\left(\mathcal{T}_{h}\right):=P^{\mathrm{g}, \mathrm{b}}\left(\mathcal{T}_{h}\right) \cap V^{\mathrm{g}}, \boldsymbol{P}^{\mathrm{c}}\left(\mathcal{T}_{h}\right):=\boldsymbol{P}^{\mathrm{c}, \mathrm{b}}\left(\mathcal{T}_{h}\right) \cap \boldsymbol{V}^{\mathrm{c}}, \boldsymbol{P}^{\mathrm{d}}\left(\mathcal{T}_{h}\right):=\boldsymbol{P}^{\mathrm{d}, \mathrm{b}}\left(\mathcal{T}_{h}\right) \cap \boldsymbol{V}^{\mathrm{d}}$; that is to say, the finite element spaces $P^{\mathrm{g}}\left(\mathcal{T}_{h}\right), \boldsymbol{P}^{\mathrm{c}}\left(\mathcal{T}_{h}\right), \boldsymbol{P}^{\mathrm{d}}\left(\mathcal{T}_{h}\right)$ are conforming in $V^{\mathrm{g}}, \boldsymbol{V}^{\mathrm{c}}, \boldsymbol{V}^{\mathrm{d}}$, respectively.

Let us introduce the canonical interpolation operators $\mathcal{I}_{h}^{\mathrm{g}}, \mathcal{I}_{h}^{\mathrm{d}}, \mathcal{I}_{h}^{\mathrm{c}}$ such that $\mathcal{I}_{h}^{\mathrm{g}}(v)_{\mid K}=\mathcal{I}_{K}^{\mathrm{g}}\left(v_{\mid K}\right), \mathcal{I}_{h}^{\mathrm{c}}(\boldsymbol{v})_{\mid K}=$ $\mathcal{I}_{K}^{\mathrm{c}}\left(\boldsymbol{v}_{\mid K}\right), \mathcal{I}_{h}^{\mathrm{d}}(\boldsymbol{v})_{\mid K}=\mathcal{I}_{K}^{\mathrm{d}}\left(\boldsymbol{v}_{\mid K}\right)$. The considerations in Section 2.4 show that it is legitimate to take $W^{s, p}(D)$, $s>\frac{d}{p}$, for the domain of $\mathcal{I}_{h}^{\mathrm{g}}, \boldsymbol{W}^{s, p}(D), s>\frac{d-1}{p}$, for the domain of $\mathcal{I}_{h}^{\mathrm{c}}$, and $\boldsymbol{W}^{s, p}(D), s>\frac{1}{p}$, for the domain of $\mathcal{I}_{h}^{\mathrm{d}}$, i.e. the canonical interpolation operators $\mathcal{I}_{h}^{\mathrm{g}}, \mathcal{I}_{h}^{\mathrm{c}}$ and $\mathcal{I}_{h}^{\mathrm{d}}$ are not stable in any $L^{p}(D)$ space (or $\boldsymbol{L}^{p}(D)$ ). The objective of this paper is to construct quasi-interpolation operators mapping onto the spaces $P^{\mathrm{g}}\left(\mathcal{T}_{h}\right), \boldsymbol{P}^{\mathrm{c}}\left(\mathcal{T}_{h}\right)$ and $\boldsymbol{P}^{\mathrm{d}}\left(\mathcal{T}_{h}\right)$ that are stable in $L^{1}(D)$ (or $\left.\boldsymbol{L}^{1}(D)\right)$ and have optimal approximation properties with and without boundary conditions.

\subsection{Summary of the assumptions}

Let us now summarize the assumptions that will be used in the rest of the paper. Henceforth $\left(\mathcal{T}_{h}\right)_{h>0}$ is a shape-regular sequence of affine, matching meshes so that (2.1) and (2.2) hold. We also assume that the map $\psi_{K}$ satisfies (2.7) and (2.9). $\left\{\left(K, P_{K}, \Sigma_{K}\right)\right\}_{K \in \mathcal{T}_{h}}$ is a $\mathcal{T}_{h}$-based sequence of finite elements constructed as in Proposition 2.1. In view of approximation, we define $k$ to be the largest natural number such that $\left[\mathbb{P}_{k, d}\right]^{q} \subset \widehat{P}$, where $\mathbb{P}_{k, d}$ is the real vector space of $d$-variate polynomials functions of degree at most $k$, and we assume that $\widehat{P} \subset W^{k+1, \infty}\left(\widehat{K} ; \mathbb{R}^{q}\right)$.

We assume that we have at hand a notion of $\gamma$-jump across mesh interfaces as described in Section 2.3. The finite element space $P\left(\mathcal{T}_{h}\right)$ is the subspace of the broken finite element space $P^{\mathrm{b}}\left(\mathcal{T}_{h}\right)$ characterized by zero $\gamma$-jumps across interfaces, see (2.17). Finally, two important assumptions relating the degrees of freedom to the $\gamma$-jump and $\gamma$-trace are the estimates (4.2) and (6.6) below.

In what follows, $c$ denotes a generic positive constant whose value may depend on the shape-regularity of the mesh sequence $\left(\mathcal{T}_{h}\right)_{h>0}$ and on the reference finite element $(\widehat{K}, \widehat{P}, \widehat{\Sigma})$. The value of this constant may vary from one occurrence to the other.

\section{3. $L^{1}$-STABLE LOCAL INTERPOLATION}

In this section we extend the degrees of freedom in order to be able to approximate functions that are only integrable.

\subsection{Extension of degrees of freedom}

Let us consider $\widehat{\rho}_{i} \in \widehat{P}, i \in \mathcal{N}$, be such that

$$
\frac{1}{|\widehat{K}|} \int_{\widehat{K}}\left(\widehat{\rho}_{i}, \widehat{p}\right)_{\ell^{2}(\mathbb{R} q)} \mathrm{d} \widehat{x}=\widehat{\sigma}_{i}(\widehat{p}), \quad \forall \widehat{p} \in \widehat{P} .
$$

Note that $\widehat{\rho}_{i}$ is well defined since it is the Riesz representative of $\widehat{\sigma}_{i}$ in $\widehat{P}$ when $\widehat{P}$ is equipped with the $L^{2}$-scalar product weighted by $1 /|\widehat{K}|$. This leads us to define

$$
\widehat{\sigma}_{i}^{\sharp}(\widehat{v}):=\frac{1}{|\widehat{K}|} \int_{\widehat{K}}\left(\widehat{\rho}_{i}, \widehat{v}\right)_{\ell^{2}\left(\mathbb{R}^{q}\right)} \mathrm{d} \widehat{x}, \quad \forall \widehat{v} \in L^{1}\left(\widehat{K} ; \mathbb{R}^{q}\right) .
$$

Note that the assumption $\widehat{P} \subset L^{\infty}\left(\widehat{K} ; \mathbb{R}^{q}\right)$ implies that $\widehat{\rho}_{i} \in L^{\infty}\left(\widehat{K} ; \mathbb{R}^{q}\right)$, which in turn implies that all the extended degrees of freedom $\left\{\widehat{\sigma}_{i}^{\sharp}\right\}_{i \in \mathcal{N}}$ are indeed bounded over $L^{1}\left(\widehat{K} ; \mathbb{R}^{q}\right)$ since $\left\|\widehat{\sigma}_{i}^{\sharp}\right\|_{\mathcal{L}\left(L^{1}\left(\widehat{K}_{;} ; \mathbb{R}^{q}\right) ; \mathbb{R}\right)} \leq$ $|\widehat{K}|^{-1}\left\|\widehat{\rho}_{i}\right\|_{L^{\infty}\left(\widehat{K} ; \mathbb{R}^{q}\right)}$. In passing we have also proved that $\left\|\widehat{\sigma}_{i}^{\sharp}\right\|_{\mathcal{L}\left(L^{p}\left(\widehat{K} ; \mathbb{R}^{q}\right) ; \mathbb{R}\right)} \leq|\widehat{K}|^{-1}\left\|\widehat{\rho}_{i}\right\|_{L^{p^{\prime}}\left(\widehat{K} ; \mathbb{R}^{q}\right)}$ for all $p \in[1, \infty]$, where $\frac{1}{p}+\frac{1}{p^{\prime}}=1$. We then define

$$
\mathcal{I}_{\widehat{K}}^{\sharp}(\widehat{v}):=\sum_{i \in \mathcal{N}} \widehat{\sigma}_{i}^{\sharp}(\widehat{v}) \widehat{\theta}_{i}, \quad \forall \widehat{v} \in L^{1}\left(\widehat{K} ; \mathbb{R}^{q}\right) .
$$

$\widehat{P}$ is point-wise invariant under $\mathcal{I}_{\widehat{K}}^{\sharp}$ since $\widehat{\sigma}_{i}^{\sharp}(\widehat{p})=\widehat{\sigma}_{i}(\widehat{p})$ for all $\widehat{p} \in \widehat{P}$ and all $i \in \mathcal{N}$. 
Let $K \in \mathcal{T}_{h}$ and let $\left(K, P_{K}, \Sigma_{K}\right)$ be a finite element constructed as in (2.5). Note that the assumption (2.7) implies that $\psi_{K}\left(L^{1}\left(K ; \mathbb{R}^{q}\right)\right)=L^{1}\left(\widehat{K} ; \mathbb{R}^{q}\right)$. We then extend the degrees of freedom in $\Sigma_{K}$ to $L^{1}\left(K ; \mathbb{R}^{q}\right)$ by setting

$$
\sigma_{K, i}^{\sharp}(v):=\widehat{\sigma}_{i}^{\sharp}\left(\psi_{K}(v)\right) .
$$

The above definition leads us to define

$$
\mathcal{I}_{K}^{\sharp}(v):=\sum_{i \in \mathcal{N}} \sigma_{K, i}^{\sharp}(v) \theta_{K, i}, \quad \forall v \in L^{1}\left(K ; \mathbb{R}^{q}\right) .
$$

Proposition 3.1 (Stability, commutation, invariance). (i) There exists a uniform constant $c$ such that $\left\|\mathcal{I}_{K}^{\sharp}\right\|_{\mathcal{L}\left(L^{p}\left(K ; \mathbb{R}^{q}\right) ; L^{p}\left(K ; \mathbb{R}^{q}\right)\right)} \leq c$, for all $p \in[1, \infty]$ and all $K \in \mathcal{T}_{h}$; (ii) $\mathcal{I}_{K}^{\sharp}$ commutes with $\psi_{K}$; (iii) $P_{K}$ is point-wise invariant under $\mathcal{I}_{K}^{\sharp}$.

Proof. Using the triangle inequality in (3.5), the fact that $\theta_{K, i}:=\psi_{K}^{-1}\left(\widehat{\theta}_{i}\right)$ and $\sigma_{K, i}^{\sharp}:=\widehat{\sigma}_{i}^{\sharp} \circ \psi_{K}$ (see (3.4)), and finally using (2.8) with $l=0$ and the assumption (2.9), we infer that

$$
\begin{aligned}
& \left\|\mathcal{I}_{K}^{\sharp}\right\|_{\mathcal{L}\left(L^{p}\left(K ; \mathbb{R}^{q}\right) ; L^{p}\left(K ; \mathbb{R}^{q}\right)\right)} \leq \sum_{i \in \mathcal{N}}\left\|\widehat{\sigma}_{i}^{\sharp}\right\|_{\mathcal{L}\left(L^{p}\left(\widehat{K} ; \mathbb{R}^{q}\right) ; \mathbb{R}\right)}\left|\psi_{K}\right|_{\mathcal{L}\left(L^{p}\left(K ; \mathbb{R}^{q}\right) ; L^{p}\left(\widehat{K} ; \mathbb{R}^{q}\right)\right)}\left\|\psi_{K}^{-1}\left(\widehat{\theta}_{i}\right)\right\|_{L^{p}\left(K ; \mathbb{R}^{q}\right)} \\
& \leq\left|\psi_{K}\right|_{\mathcal{L}\left(L^{p}\left(K ; \mathbb{R}^{q}\right) ; L^{p}\left(\widehat{K} ; \mathbb{R}^{q}\right)\right)}\left|\psi_{K}^{-1}\right|_{\mathcal{L}\left(L^{p}\left(\widehat{K} ; \mathbb{R}^{q}\right) ; L^{p}\left(K ; \mathbb{R}^{q}\right)\right)} \sum_{i \in \mathcal{N}}\left\|\widehat{\sigma}_{i}^{\sharp}\right\|_{\mathcal{L}\left(L^{p}\left(\widehat{K} ; \mathbb{R}^{q}\right) ; \mathbb{R}\right)}\left\|\widehat{\theta}_{i}\right\|_{L^{p}\left(\widehat{K} ; \mathbb{R}^{q}\right)} \\
& \leq c\left\|\mathbb{A}_{K}\right\|_{\ell^{2}}\left\|\mathbb{A}_{K}^{-1}\right\|_{\ell^{2}} \sum_{i \in \mathcal{N}}\left\|\widehat{\sigma}_{i}^{\sharp}\right\|_{\mathcal{L}\left(L^{p}\left(\widehat{K} ; \mathbb{R}^{q}\right) ; \mathbb{R}\right)}\left\|\widehat{\theta}_{i}\right\|_{L^{p}\left(\widehat{K} ; \mathbb{R}^{q}\right)} \\
& \leq c\left\|\mathbb{J}_{K}\right\|_{\ell^{2}}\left\|\mathbb{J}_{K}^{-1}\right\|_{\ell^{2}}|\widehat{K}|^{-1} \sum_{i \in \mathcal{N}}\left\|\widehat{\rho}_{i}\right\|_{L^{p^{\prime}}\left(\widehat{K} ; \mathbb{R}^{q}\right)} .
\end{aligned}
$$

The conclusion readily follows from the shape-regularity assumptions. To prove the second statement, we use again that $\theta_{K, i}=\psi_{K}^{-1}\left(\widehat{\theta}_{i}\right)$ to infer that

$$
\psi_{K}\left(\mathcal{I}_{K}^{\sharp}(v)\right):=\psi_{K}\left(\sum_{i \in \mathcal{N}} \sigma_{K, i}^{\sharp}(v) \psi_{K}^{-1}\left(\widehat{\theta}_{i}\right)\right)=\sum_{i \in \mathcal{N}} \widehat{\sigma}_{i}^{\sharp}\left(\psi_{K}(v)\right) \widehat{\theta}_{i}=\mathcal{I}_{\widehat{K}}^{\sharp}\left(\psi_{K}(v)\right),
$$

for all $v \in L^{1}\left(K ; \mathbb{R}^{q}\right)$. To prove the third statement, let us consider any $g \in P_{K}=\psi_{K}(\widehat{P})$; then using the above definitions we have

$$
\sigma_{K, i}^{\sharp}(g)=\widehat{\sigma}_{i}^{\sharp}\left(\psi_{K}(g)\right)=\frac{1}{|\widehat{K}|} \int_{\widehat{K}}\left(\widehat{\rho}_{i}, \psi_{K}(g)\right)_{\ell^{2}\left(\mathbb{R}^{q}\right)} \mathrm{d} \widehat{x}=\widehat{\sigma}_{i}\left(\psi_{K}(g)\right)=\sigma_{K, i}(g) .
$$

This proves that $\mathcal{I}_{K}^{\sharp}(g)=\mathcal{I}_{K}(g)$, hence $\mathcal{I}_{K}^{\sharp}(g)=g$.

Remark 3.2. The construction of $\mathcal{I}_{K}^{\sharp}$ is similar to that used in (Girault and Lions [15], Appendix) to extend the Scott-Zhang quasi-interpolation operator and make it stable in $L^{1}(D)$ for scalar-valued functions.

\subsection{Error estimates for $\mathcal{I}_{K}^{\sharp}$}

We establish in this section error estimates for the operator $\mathcal{I}_{K}^{\sharp}$.

Theorem 3.3 (Local interpolation). There exists a uniform constant c such that the following local error estimate holds:

$$
\left|v-\mathcal{I}_{K}^{\sharp} v\right|_{W^{m, p}\left(K ; \mathbb{R}^{q}\right)} \leq c h_{K}^{l-m}|v|_{W^{l, p}\left(K ; \mathbb{R}^{q}\right)},
$$

for all $l \in\{0: k+1\}$, all $m \in\{0: l\}$, all $p \in[1, \infty]$, all $v \in W^{l, p}\left(K ; \mathbb{R}^{q}\right)$, and all $K \in \mathcal{T}_{h}$. 
Proof. Let $l \in\{0: k+1\}$ and $m \in\{0: l\}$.

(1) Let us set $\mathcal{G}(\widehat{w}):=\widehat{w}-\mathcal{I}_{\widehat{K}}^{\sharp}(\widehat{w})$ for all $\widehat{w} \in W^{l, p}\left(\widehat{K} ; \mathbb{R}^{q}\right)$. The operator $\mathcal{G}$ is well-defined since $W^{l, p}\left(\widehat{K} ; \mathbb{R}^{q}\right) \hookrightarrow$ $L^{1}\left(\widehat{K} ; \mathbb{R}^{q}\right)$. Since all the norms are equivalent in $\widehat{P}$ and $\widehat{P} \subset W^{k+1, p}\left(\widehat{K} ; \mathbb{R}^{q}\right) \hookrightarrow W^{m, p}\left(\widehat{K} ; \mathbb{R}^{q}\right)$, there exists $c$ depending only on $n_{\text {sh }}$ and $\widehat{K}$ such that $\left\|\mathcal{I}_{\widehat{K}}^{\sharp}(\widehat{w})\right\|_{W^{m, p}\left(\widehat{K} ; \mathbb{R}^{q}\right)} \leq c\left\|\mathcal{I}_{\widehat{K}}^{\sharp}(\widehat{w})\right\|_{L^{1}\left(\widehat{K} ; \mathbb{R}^{q}\right)}$, which in turns implies that $\left\|\mathcal{I}_{\widehat{K}}^{\sharp}(\widehat{w})\right\|_{W^{m, p}\left(\widehat{K} ; \mathbb{R}^{q}\right)} \leq c\|\widehat{w}\|_{L^{1}(\widehat{K} ; \mathbb{R} q)}$, since we have already established that $\mathcal{I}_{\widehat{K}}^{\sharp}$ is uniformly bounded over $L^{1}\left(\widehat{K} ; \mathbb{R}^{q}\right)$; hence, $\mathcal{G} \in \mathcal{L}\left(W^{l, p}\left(\widehat{K} ; \mathbb{R}^{q}\right) ; W^{m, p}\left(\widehat{K} ; \mathbb{R}^{q}\right)\right)$. Assume first that $l \geq 1$, then $\left[\mathbb{P}_{l-1}\right]^{q}$ is pointwise invariant under $\mathcal{I}_{\widehat{K}}^{\sharp}$ since $l-1 \leq k$ and $\left[\mathbb{P}_{l-1}\right]^{q} \subset\left[\mathbb{P}_{k}\right]^{q} \subset \widehat{P}$; this in turn implies that the operator $\mathcal{G}$ vanishes on $\left[\mathbb{P}_{l-1}\right]^{q}$. As a consequence, we infer that

$$
\begin{aligned}
\left|\widehat{w}-\mathcal{I}_{\widehat{K}}^{\sharp} \widehat{w}\right|_{W^{m, p}\left(\widehat{K} ; \mathbb{R}^{q}\right)} & =|\mathcal{G}(\widehat{w})|_{W^{m, p}\left(\widehat{K} ; \mathbb{R}^{q}\right)}=\inf _{\widehat{p} \in\left[\mathbb{P}_{l-1}\right]^{q}}|\mathcal{G}(\widehat{w}+\widehat{p})|_{W^{m, p}\left(\widehat{K} ; \mathbb{R}^{q}\right)} \\
& \leq\|\mathcal{G}\|_{\mathcal{L}\left(W^{l, p}\left(\widehat{K} ; \mathbb{R}^{q}\right) ; W^{m, p}\left(\widehat{K} ; \mathbb{R}^{q}\right)\right)} \inf _{\widehat{p} \in\left[\mathbb{P}_{l-1}\right]^{q}}\|\widehat{w}+\widehat{p}\|_{W^{l, p}\left(\widehat{K} ; \mathbb{R}^{q}\right)} \\
& \leq c \inf _{\widehat{p} \in\left[\mathbb{P}_{l-1}\right]^{q}}\|\widehat{w}+\widehat{p}\|_{W^{l, p}\left(\widehat{K} ; \mathbb{R}^{q}\right)} \leq c|\widehat{w}|_{W^{l, p}\left(\widehat{K} ; \mathbb{R}^{q}\right)},
\end{aligned}
$$

for all $\widehat{w} \in W^{l, p}\left(\widehat{K} ; \mathbb{R}^{q}\right)$, where the last estimate is a consequence of the Bramble-Hilbert/Deny-Lions Lemma. Finally, the above inequality is trivial if $l=m=0$.

(2) Now let $v \in W^{l, p}\left(K ; \mathbb{R}^{q}\right)$. Using the above argument together with the fact that $\mathcal{I}_{K}^{\sharp}$ commutes with $\psi_{K}$ (see Prop. 3.1), we have

$$
\begin{aligned}
\left|v-\mathcal{I}_{K}^{\sharp} v\right|_{W^{m, p}\left(K ; \mathbb{R}^{q}\right)} & \leq\left|\psi_{K}^{-1}\right|_{\mathcal{L}\left(W^{m, p}\left(\widehat{K} ; \mathbb{R}^{q}\right) ; W^{m, p}\left(K ; \mathbb{R}^{q}\right)\right)}\left|\psi_{K}(v)-\psi_{K}\left(\mathcal{I}_{K}^{\sharp} v\right)\right|_{W^{m, p}\left(\widehat{K} ; \mathbb{R}^{q}\right)} \\
& \leq\left|\psi_{K}^{-1}\right|_{\mathcal{L}\left(W^{m, p}\left(\widehat{K} ; \mathbb{R}^{q}\right) ; W^{m, p}\left(K ; \mathbb{R}^{q}\right)\right)}\left|\psi_{K}(v)-\mathcal{I}_{\widehat{K}}^{\sharp}\left(\psi_{K}(v)\right)\right|_{W^{m, p}\left(\widehat{K} ; \mathbb{R}^{q}\right)} \\
& \leq c\left|\psi_{K}^{-1}\right|_{\mathcal{L}\left(W^{m, p}\left(\widehat{K} ; \mathbb{R}^{q}\right) ; W^{m, p}\left(K ; \mathbb{R}^{q}\right)\right)}\left|\psi_{K}(v)\right|_{W^{l, p}\left(\widehat{K} ; \mathbb{R}^{q}\right)} \\
& \leq c\left|\psi_{K}^{-1}\right|_{\mathcal{L}\left(W^{m, p}\left(\widehat{K} ; \mathbb{R}^{q}\right) ; W^{m, p}\left(K ; \mathbb{R}^{q}\right)\right)}\left|\psi_{K}\right|_{\mathcal{L}\left(W^{l, p}\left(K ; \mathbb{R}^{q}\right) ; W^{l, p}\left(\widehat{K} ; \mathbb{R}^{q}\right)\right)}|v|_{W^{l, p}\left(K ; \mathbb{R}^{q}\right)} .
\end{aligned}
$$

The estimate (3.6) follows by using (2.10).

\section{Averaging operator}

In this section, we introduce a bounded linear operator $\mathcal{J}_{h}^{\text {av }}: P^{\mathrm{b}}\left(\mathcal{T}_{h}\right) \rightarrow P\left(\mathcal{T}_{h}\right)$ based on averaging.

\subsection{Connectivity array}

Let $\left\{\varphi_{a}\right\}_{a \in \mathcal{A}_{h}}$ be a basis of $P\left(\mathcal{T}_{h}\right)$; the functions $\varphi_{a}$ are called global shape functions. We assume that this basis is constructed so that for any $K \in \mathcal{T}_{h}$, either $\operatorname{int}(K) \cap \operatorname{supp}\left(\varphi_{a}\right)=\emptyset$ or there is a unique $i \in \mathcal{N}$ such that $\varphi_{a \mid K}=\theta_{K, i}$. (Recall that this is the usual way of constructing finite element bases.) We denote by a $: \mathcal{T}_{h} \times \mathcal{N} \rightarrow \mathcal{A}_{h}$ the map such that $\varphi_{\mathrm{a}(K, i) \mid K}=\theta_{K, i}$; this map is henceforth called connectivity array. Note that a is surjective by definition, but in general a is not injective. Denoting by a ${ }^{-1}(a)$ the preimage of $a \in \mathcal{A}_{h}$, we define the connectivity set $\mathcal{C}_{a} \subset \mathcal{T}_{h} \times \mathcal{N}$ for any $a \in \mathcal{A}_{h}$ such that

$$
\mathcal{C}_{a}:=\mathrm{a}^{-1}(a)=\left\{(K, i) \in \mathcal{T}_{h} \times \mathcal{N} \mid a=\mathrm{a}(K, i)\right\} .
$$

Remark 4.1 (Particular case $\operatorname{card}\left(\mathcal{C}_{a}\right)=1$ ). Assume that $\operatorname{card}\left(\mathcal{C}_{a}\right)=1$, i.e. $\mathcal{C}_{a}=\left\{\left(K_{0}, i_{0}\right)\right\}$. Let $K \neq K_{0}$, then it is not possible to find an index $i \in \mathcal{N}$ such that $a=\mathrm{a}(K, i) \operatorname{since} \operatorname{card}\left(\mathcal{C}_{a}\right)=1$; this in turn implies that $\operatorname{int}(K) \cap \operatorname{supp}\left(\varphi_{a}\right)=\emptyset$, hence $\varphi_{a \mid} \operatorname{int}(K)=0$. This means that $\varphi_{a}$ is supported on one element only, i.e. $\varphi_{a}$ is the zero extension of $\theta_{K_{0}, i_{0}}$. Given the characterization of $P\left(\mathcal{T}_{h}\right)$ assumed in (2.17), this means that the $\gamma$-trace of $\varphi_{a}$ on the interior faces of $K$ is zero. 
For any $a \in \mathcal{A}_{h}$, we set $\mathcal{F}_{a}^{\circ}=\emptyset$ if $\operatorname{card}\left(\mathcal{C}_{a}\right)=1$. If $\operatorname{card}\left(\mathcal{C}_{a}\right) \geq 2$ we define $\mathcal{F}_{a}^{\circ} \subset \mathcal{F}_{h}^{\circ}$ to be the set of the interfaces $F$ such that there are $(K, i),\left(K^{\prime}, i^{\prime}\right) \in \mathcal{C}_{a}$ so that $F=\partial K \cap \partial K^{\prime}$. We now relate the $\gamma$-traces to the degrees of freedom by making the following assumption: there exists a uniform constant $c$ such that the following holds for all $v$ in $P^{\mathrm{b}}\left(\mathcal{T}_{h}\right)$ and all $a \in \mathcal{A}_{h}$ such that $\operatorname{card}\left(\mathcal{C}_{a}\right) \geq 2$ :

$$
\left|\sigma_{K, i}\left(v_{\mid K}\right)-\sigma_{K^{\prime}, i^{\prime}}\left(v_{\mid K^{\prime}}\right)\right| \leq c \min \left(\left\|\mathbb{A}_{K}\right\|_{\ell^{2}},\left\|\mathbb{A}_{K^{\prime}}\right\|_{\ell^{2}}\right)\left\|\llbracket v \rrbracket_{F}^{\gamma}\right\|_{L^{\infty}\left(F ; \mathbb{R}^{t}\right)},
$$

for all $F \in \mathcal{F}_{a}^{\circ}$ and all pairs $(K, i),\left(K^{\prime}, i^{\prime}\right) \in \mathcal{C}_{a}$ such that $F=\partial K \cap \partial K^{\prime}$. The estimate (4.2) is natural since the degrees of freedom of finite elements providing some conformity in $H^{1}, \boldsymbol{H}$ (curl), or $\boldsymbol{H}$ (div) are devised to coincide across interfaces when the $\gamma$-jump is zero and when the degrees of freedom in question belong to the same connectivity class. In particular, the estimate (4.2) holds true for all the finite elements considered in Section 2.4. Owing to (2.17), the assumption (4.2) immediately implies that

$$
\left|\sigma_{K, i}\left(v_{\mid K}\right)-\sigma_{K^{\prime}, i^{\prime}}\left(v_{\mid K^{\prime}}\right)\right|=0, \quad \forall v \in P\left(\mathcal{T}_{h}\right), \forall a \in \mathcal{A}_{h}, \forall(K, i),\left(K^{\prime}, i^{\prime}\right) \in \mathcal{C}_{a} .
$$

\subsection{Averaging operator}

We define the operator $\mathcal{J}_{h}^{\text {av }}: P^{\mathrm{b}}\left(\mathcal{T}_{h}\right) \rightarrow P\left(\mathcal{T}_{h}\right)$ by

$$
\mathcal{J}_{h}^{\mathrm{av}}(v)=\sum_{a \in \mathcal{A}_{h}}\left(\frac{1}{\operatorname{card}\left(\mathcal{C}_{a}\right)} \sum_{(K, i) \in \mathcal{C}_{a}} \sigma_{K, i}\left(v_{\mid K}\right)\right) \varphi_{a} .
$$

For any $K \in \mathcal{T}_{h}$, we introduce the notation

$$
\begin{aligned}
\mathcal{T}_{K} & :=\cup_{i \in \mathcal{N}}\left\{K^{\prime} \in \mathcal{T}_{h} \mid \exists i^{\prime} \in \mathcal{N},\left(K^{\prime}, i^{\prime}\right) \in \mathcal{C}_{\mathrm{a}(K, i)}\right\}, \\
D_{K} & :=\operatorname{int}\left\{\cup_{K^{\prime} \in \mathcal{T}_{K}} K^{\prime}\right\} .
\end{aligned}
$$

The set $\mathcal{T}_{K}$ is the union of all the cells that share global shape functions with $K$ and $D_{K}$ is the interior of the collection of the points composing the cells in $\mathcal{T}_{K}$.

Lemma 4.2 ( $L^{p}$-stability). There exists a uniform constant $c$ such that

$$
\left\|\mathcal{J}_{h}^{\text {av }}(v)\right\|_{L^{p}\left(K ; \mathbb{R}^{q}\right)} \leq c\|v\|_{L^{p}\left(D_{K} ; \mathbb{R}^{q}\right)},
$$

for all $p \in[1, \infty]$, all $v \in P^{\mathrm{b}}\left(\mathcal{T}_{h}\right)$, and all $K \in \mathcal{T}_{h}$.

Proof. We prove the result for $p=\infty$; the other cases are obtained by using local inverse inequalities in $P^{\mathrm{b}}\left(\mathcal{T}_{h}\right)$. Using the triangle inequality and the shape-regularity of the mesh sequence $\left(\mathcal{T}_{h}\right)_{h>0}$, we infer that

$$
\begin{aligned}
\left\|\mathcal{J}_{h}^{\mathrm{av}}(v)\right\|_{L^{\infty}\left(K ; \mathbb{R}^{q}\right)} & \leq \sum_{i \in \mathcal{N}} \frac{\left\|\theta_{K, i}\right\|_{L^{\infty}\left(K ; \mathbb{R}^{q}\right)}}{\operatorname{card}\left(\mathcal{C}_{\mathrm{a}(K, i)}\right)} \sum_{\left(K^{\prime}, i^{\prime}\right) \in \mathcal{C}_{\mathrm{a}(K, i)}}\left|\sigma_{K^{\prime}, i^{\prime}}\left(v_{\mid K^{\prime}}\right)\right| \\
& \leq c \sum_{i \in \mathcal{N}} \frac{\left\|\mathbb{A}_{K}^{-1}\right\|_{\ell^{2}}}{\operatorname{card}\left(\mathcal{C}_{\mathrm{a}(K, i)}\right)} \sum_{\left(K^{\prime}, i^{\prime}\right) \in \mathcal{C}_{\mathrm{a}(K, i)}}\left|\sigma_{K^{\prime}, i^{\prime}}\left(v_{\mid K^{\prime}}\right)\right| \\
& \leq c \sum_{K^{\prime} \in \mathcal{T}_{K}}\left\|\mathbb{A}_{K}^{-1}\right\|_{\ell^{2}} \sum_{i^{\prime} \in \mathcal{N}}\left|\sigma_{K^{\prime}, i^{\prime}}\left(v_{\mid K^{\prime}}\right)\right| \leq c\|v\|_{L^{\infty}\left(D_{K} ; \mathbb{R}^{q}\right)},
\end{aligned}
$$

where the last estimate results from $\left|\sigma_{K^{\prime}, i^{\prime}}\left(v_{\mid K^{\prime}}\right)\right| \leq\left|\sigma_{K^{\prime}, i^{\prime}}\left(v_{\mid K^{\prime}}\right)-\sigma_{K, i}\left(v_{\mid K}\right)\right|+\left|\sigma_{K, i}\left(v_{\mid K}\right)\right|$, the assumption (4.2), the inequality $\left|\llbracket v \rrbracket_{F}^{\gamma}\right|_{L^{\infty}\left(F ; \mathbb{R}^{t}\right)} \leq\left|\llbracket v \rrbracket_{F}\right|_{L^{\infty}\left(F ; \mathbb{R}^{t}\right)} \leq\|v\|_{L^{\infty}\left(D_{K} ; \mathbb{R}^{q}\right)}$ and $(2.12)$. 
Lemma 4.3 (Approximation by averaging). There exists a uniform constant $c$ such that the following holds with the notation $\mathcal{F}_{K}^{\circ}:=\cup_{i \in \mathcal{N}} \mathcal{F}_{\mathrm{a}(K, i)}^{\circ}$ :

$$
\left|v-\mathcal{J}_{h}^{\mathrm{av}}(v)\right|_{W^{m, p}\left(K ; \mathbb{R}^{q}\right)} \leq \operatorname{ch}_{K}^{d\left(\frac{1}{p}-\frac{1}{r}\right)+\frac{1}{r}-m} \sum_{F \in \mathcal{F}_{K}^{\circ}}\left\|\llbracket v \rrbracket_{F}^{\gamma}\right\|_{L^{r}\left(F ; \mathbb{R}^{t}\right)},
$$

for all $m \in\{0: k+1\}$, all $p, r \in[1, \infty]$, all $v \in P^{\mathrm{b}}\left(\mathcal{T}_{h}\right)$, and all $K \in \mathcal{T}_{h}$.

Proof. We only prove the bound for $m=0$ and $p=r=\infty$, the other cases follow by invoking standard inverse inequalities. Let $v \in P^{\mathrm{b}}\left(\mathcal{T}_{h}\right)$, set $e=v-\mathcal{J}_{h}^{\text {av }}(v)$ and observe that $e \in P^{\mathrm{b}}\left(\mathcal{T}_{h}\right)$. Let $K \in \mathcal{T}_{h}$. The bound $(2.11)$ implies that

$$
\|e\|_{L^{\infty}\left(K ; \mathbb{R}^{q}\right)} \leq c\left\|\mathbb{A}_{K}^{-1}\right\|_{\ell^{2}} \sum_{i \in \mathcal{N}}\left|\sigma_{K, i}\left(e_{\mid K}\right)\right| .
$$

Owing to the definition of $\mathcal{J}_{h}^{\text {av }}$ in (4.4), we first observe that

$$
\sigma_{K, i}\left(e_{\mid K}\right)=\frac{1}{\operatorname{card}\left(\mathcal{C}_{\mathrm{a}(K, i)}\right)} \sum_{\left(K^{\prime}, i^{\prime}\right) \in \mathcal{C}_{\mathrm{a}(K, i)}}\left(\sigma_{K, i}\left(v_{\mid K}\right)-\sigma_{K^{\prime}, i^{\prime}}\left(v_{\mid K^{\prime}}\right)\right) .
$$

Note that $\sigma_{K, i}\left(e_{\mid K}\right)=0$ if $\operatorname{card}\left(\mathcal{C}_{\mathrm{a}(K, i)}\right)=1$ (see Rem. 4.1). Let us now consider the case $\operatorname{card}\left(\mathcal{C}_{\mathrm{a}(K, i)}\right) \geq 2$. For all $\left(K^{\prime}, i^{\prime}\right) \in \mathcal{C}_{\mathrm{a}(K, i)}$, there is a path of mesh cells in $\mathcal{T}_{K}$ linking $K$ to $K^{\prime}$ so that any two consecutive mesh cells in the path share a common face $F \in \mathcal{F}_{\mathrm{a}(K, i)}^{\circ}$, and each face is crossed only once. Furthermore, if $\left(K_{l}, i_{l}\right),\left(K_{r}, i_{r}\right) \in \mathcal{C}_{\mathrm{a}(K, i)}$ are such that $\partial K_{l} \cap \partial K_{r}=F \in \mathcal{F}_{\mathrm{a}(K, i)}^{\circ}$, then (4.2) implies that

$$
\left|\sigma_{K_{l}, i_{l}}\left(v_{\mid K_{l}}\right)-\sigma_{K_{r}, i_{r}}\left(v_{\mid K_{r}}\right)\right| \leq c \min \left(\left\|\mathbb{A}_{K_{l}}\right\|_{\ell^{2}},\left\|\mathbb{A}_{K_{r}}\right\|_{\ell^{2}}\right)\left\|\llbracket v \rrbracket_{F}^{\gamma}\right\|_{L^{\infty}\left(F ; \mathbb{R}^{t}\right)} .
$$

As a result,

$$
\|e\|_{L^{\infty}\left(K ; \mathbb{R}^{q}\right)} \leq c\left\|\mathbb{A}_{K}^{-1}\right\|_{\ell^{2}}\left\|\mathbb{A}_{K}\right\|_{\ell^{2}} \sum_{F \in \mathcal{F}_{a(K, i)}^{\circ}}\|\llbracket v\|_{F}^{\gamma} \|_{L^{\infty}\left(F ; \mathbb{R}^{t}\right)},
$$

whence the estimate (4.7) readily follows since $\mathcal{F}_{K}^{\circ}:=\cup_{i \in \mathcal{N}} \mathcal{F}_{\mathrm{a}(K, i)}^{\circ}$, $\operatorname{card}(\mathcal{N})$ is uniformly bounded, and the mesh sequence $\left(\mathcal{T}_{h}\right)_{h>0}$ is shape-regular.

\section{Quasi-INTERPolation OPERATOR}

Let $\mathcal{I}_{h}^{\sharp}: L^{1}\left(D ; \mathbb{R}^{q}\right) \rightarrow P^{\mathrm{b}}\left(\mathcal{T}_{h}\right)$ be such that $\mathcal{I}_{h}^{\sharp}(v)_{\mid K}=\mathcal{I}_{K}^{\sharp}\left(v_{\mid K}\right)$ for all $K \in \mathcal{T}_{h}$. We now construct the global quasi-interpolation operator $\mathcal{I}_{h}^{\text {av }}: L^{1}\left(D ; \mathbb{R}^{q}\right) \rightarrow P\left(\mathcal{T}_{h}\right)$ by setting

$$
\mathcal{I}_{h}^{\text {av }}:=\mathcal{J}_{h}^{\text {av }} \circ \mathcal{I}_{h}^{\sharp} \text {. }
$$

Note that $P\left(\mathcal{T}_{h}\right)$ is point-wise invariant under $\mathcal{I}_{h}^{\text {av }}$ since $P\left(\mathcal{T}_{h}\right)$ is point-wise invariant under $\mathcal{J}_{h}^{\text {av }}$ and $\mathcal{I}_{h}^{\sharp}$. Hence, $\mathcal{I}_{h}^{\text {av }}$ is a projection, i.e. $\left(\mathcal{I}_{h}^{\text {av }}\right)^{2}=\mathcal{I}_{h}^{\text {av }}$.

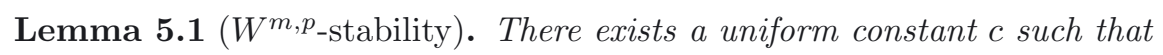

$$
\left|\mathcal{I}_{h}^{\mathrm{av}}(v)\right|_{W^{m, p}\left(K ; \mathbb{R}^{q}\right)} \leq c|v|_{W^{m, p}\left(D_{K} ; \mathbb{R}^{q}\right)},
$$

for all $p \in[1, \infty]$, all $m \in\{0: k+1\}$, all $v \in W^{m, p}\left(D_{K} ; \mathbb{R}^{q}\right)$, and all $K \in \mathcal{T}_{h}$. 
Proof. For $m=0$, the estimate follows by combining Proposition 3.1 with Lemma 4.2. For $m \geq 1$, the triangle inequality implies that

$$
\left|\mathcal{I}_{h}^{\mathrm{av}}(v)\right|_{W^{m, p}\left(K ; \mathbb{R}^{q}\right)} \leq\left|\mathcal{I}_{K}^{\sharp}(v)\right|_{W^{m, p}\left(K ; \mathbb{R}^{q}\right)}+\left|\mathcal{I}_{K}^{\sharp}(v)-\mathcal{J}_{h}^{\mathrm{av}}\left(\mathcal{I}_{K}^{\sharp}(v)\right)\right|_{W^{m, p}\left(K ; \mathbb{R}^{q}\right)} .
$$

Let $\mathfrak{T}_{1}$ and $\mathfrak{T}_{2}$ be the two terms on the right-hand side of the above inequality. $\mathfrak{T}_{1}$ is estimated using Theorem 3.3 with $l=m$, yielding $\left|\mathfrak{T}_{1}\right| \leq c|v|_{W^{m, p}\left(K ; \mathbb{R}^{q}\right)}$. $\mathfrak{T}_{2}$ is estimated using Lemma 4.3 and the fact that $v \in W^{m, p}\left(D_{K} ; \mathbb{R}^{q}\right) \subset W^{1,1}\left(D_{K} ; \mathbb{R}^{q}\right)$ has zero $\gamma$-jumps across interfaces (see (2.16)). More precisely, we have

$$
\begin{aligned}
h_{K}^{m}\left|\mathfrak{T}_{2}\right| & \leq \operatorname{ch} \frac{1}{p} \sum_{F \in \mathcal{F}_{K}^{\circ}}\left\|\llbracket \mathcal{I}_{K}^{\sharp}(v) \rrbracket_{F}^{\gamma}\right\|_{L^{p}\left(F ; \mathbb{R}^{t}\right)}=c h_{K}^{\frac{1}{p}} \sum_{F \in \mathcal{F}_{K}^{\circ}}\left\|\llbracket v-\mathcal{I}_{K}^{\sharp}(v) \rrbracket_{F}^{\gamma}\right\|_{L^{p}\left(F ; \mathbb{R}^{t}\right)} \\
& \leq \operatorname{ch}_{K}^{\frac{1}{p}} \sum_{K^{\prime} \in \mathcal{T}_{K}} \sum_{F \subset \partial K^{\prime} \cap \mathcal{F}_{K}^{\circ}}\left\|\left(v-\mathcal{I}_{K}^{\sharp}(v)\right)_{\mid K^{\prime}}\right\|_{L^{p}\left(F ; \mathbb{R}^{q}\right)} \leq c h_{K}^{m} \sum_{K^{\prime} \in \mathcal{T}_{K}}|v|_{W^{m, p}\left(K^{\prime} ; \mathbb{R}^{q}\right)},
\end{aligned}
$$

where we have used the triangle inequality to bound the jump by the values over the two adjacent mesh cells, the trace inequality from Lemma 7.2 with $s=1$, the approximation result of Theorem 3.3, and the shape regularity of the mesh sequence. Combining the bounds on $\mathfrak{T}_{1}$ and $\mathfrak{T}_{2}$ gives (5.2).

Let us now estimate the approximation properties of $\mathcal{I}_{h}^{\text {av }}$. Since we are going to establish error estimates in fractional Sobolev spaces, in the rest of the paper all the results that are stated in fractional Sobolev spaces assume that $p \in[1, \infty)$, whereas the results with integer degree of smoothness hold for $p \in[1, \infty]$. Assuming that $r \notin \mathbb{N}$ and denoting by $\lfloor r\rfloor$ the largest integer less than or equal to $r$, we consider the so-called Sobolev-Slobodeckij norm defined as $\|v\|_{W^{r, p}\left(D ; \mathbb{R}^{q}\right)}=\left(\|v\|_{W{ }^{\lfloor r}, p\left(D ; \mathbb{R}^{q}\right)}^{p}+|v|_{W^{r, p}\left(D ; \mathbb{R}^{q}\right)}^{p}\right)^{\frac{1}{p}}$ with

$$
|v|_{W^{r, p}\left(D ; \mathbb{R}^{q}\right)}=\left(\sum_{|\alpha|=\lfloor r\rfloor} \int_{D} \int_{D} \frac{\left\|\partial^{\alpha} v(\boldsymbol{x})-\partial^{\alpha} v(\boldsymbol{y})\right\|_{\ell^{2}\left(\mathbb{R}^{q}\right)}^{p}}{\|\boldsymbol{x}-\boldsymbol{y}\|_{\ell^{2}\left(\mathbb{R}^{d}\right)}^{(r-\lfloor r\rfloor)+d}} \mathrm{~d} x \mathrm{~d} y\right)^{\frac{1}{p}} .
$$

Theorem 5.2 (Local approximation). There exists a uniform constant $c$ such that

$$
\left|v-\mathcal{I}_{h}^{\mathrm{av}}(v)\right|_{W^{m, p}\left(K ; \mathbb{R}^{q}\right)} \leq c h_{K}^{r-m}|v|_{W^{r, p}\left(D_{K} ; \mathbb{R}^{q}\right)},
$$

for all $r \in[0, k+1]$, all $m \in\{0:\lfloor r\rfloor\}$, all $p \in[1, \infty)$ if $r \notin \mathbb{N}$ or all $p \in[1, \infty]$ if $r \in \mathbb{N}$, all $v \in W^{r, p}\left(D_{K} ; \mathbb{R}^{q}\right)$, and all $K \in \mathcal{T}_{h}$.

Proof. Using that $\mathcal{I}_{h}^{\text {av }}(g)=g$ for all $g \in\left[\mathbb{P}_{k, d}\right]^{q}$ together with the stability of $\mathcal{I}_{h}^{\text {av }}$ in the $W^{m, p_{\text {-seminorm }} \text { and }}$ the triangle inequality, we infer that

$$
\begin{aligned}
\left|v-\mathcal{I}_{h}^{\mathrm{av}}(v)\right|_{W^{m, p}\left(K ; \mathbb{R}^{q}\right)} & =\left|v-g-\mathcal{I}_{h}^{\mathrm{av}}(v-g)\right|_{W^{m, p}\left(K ; \mathbb{R}^{q}\right)} \\
& \leq|v-g|_{W^{m, p}\left(K ; \mathbb{R}^{q}\right)}+\left|\mathcal{I}_{h}^{\mathrm{av}}(v-g)\right|_{W^{m, p}\left(K ; \mathbb{R}^{q}\right)} \\
& \leq c|v-g|_{W^{m, p}\left(D_{K} ; \mathbb{R}^{q}\right) .}
\end{aligned}
$$

That is to say, $\left|v-\mathcal{I}_{h}^{\text {av }}(v)\right|_{W^{m, p}\left(K ; \mathbb{R}^{q}\right)} \leq c \inf _{g \in\left[\mathbb{P}_{k, d}\right]^{q}}|v-g|_{W^{m, p}\left(D_{K} ; \mathbb{R}^{q}\right)}$. We conclude by applying Lemma 5.6 below componentwise.

Corollary 5.3 (Global best approximation in $L^{p}$ ). There exists a uniform constant $c$ such that

$$
\inf _{w_{h} \in P\left(\mathcal{T}_{h}\right)}\left\|v-w_{h}\right\|_{L^{p}\left(D ; \mathbb{R}^{q}\right)} \leq c h^{r}|v|_{W^{r, p}\left(D ; \mathbb{R}^{q}\right)},
$$

for all $r \in[0, k+1]$, all $p \in[1, \infty)$ if $r \notin \mathbb{N}$ or all $p \in[1, \infty]$ if $r \in \mathbb{N}$, and all $v \in W^{r, p}\left(D ; \mathbb{R}^{q}\right)$. 
Remark 5.4 (Interpolation). Corollary 5.3 can also be proved using (5.4) for $r \in \mathbb{N}$ and the real interpolation method (i.e. the $K$-method), see e.g. (Tartar [24], Chap. 22).

Remark 5.5 (Approximation for $\mathcal{I}_{K}^{\sharp}$ ). Note in passing that Theorem 3.3, which states the approximation properties of $\mathcal{I}_{K}^{\sharp}$, can be re-written with fractional Sobolev norms, i.e. the following also holds:

$$
\left|v-\mathcal{I}_{K}^{\sharp}(v)\right|_{W^{m, p}\left(K ; \mathbb{R}^{q}\right)} \leq c h_{K}^{r-m}|v|_{W^{r, p}\left(K ; \mathbb{R}^{q}\right)},
$$

for all $r \in[0, k+1]$, all $m \in\{0:\lfloor r\rfloor\}$, all $p \in[1, \infty)$ if $r \notin \mathbb{N}$ or all $p \in[1, \infty]$ if $r \in \mathbb{N}$, all $v \in W^{r, p}\left(K ; \mathbb{R}^{q}\right)$, and all $K \in \mathcal{T}_{h}$.

Lemma 5.6 (Polynomial approximation in $D_{K}$ ). The following holds:

$$
\inf _{g \in \mathbb{P}_{k, d}}|v-g|_{W^{m, p}\left(D_{K}\right)} \leq c h_{K}^{r-m}|v|_{W^{r, p}\left(D_{K}\right)}
$$

for all $r \in[0, k+1]$, all $m \in\{0:\lfloor r\rfloor\}$, all $p \in[1, \infty)$ if $r \notin \mathbb{N}$ or all $p \in[1, \infty]$ if $r \in \mathbb{N}$, all $v \in W^{r, p}\left(D_{K}\right)$, and all $K \in \mathcal{T}_{h}$.

Proof. We proceed as in (Bramble and Hilbert [4], Thm. 1), but instead of invoking (Morrey [19], Thm. 3.6.11), where the constants may depend on $D_{K}$, we are going to track the constants to make sure that they are independent of $D_{K}$. If $m=r$, there is nothing to prove. Let us assume that $m<r$. Let $\ell \in \mathbb{N}$ be such that $\ell=r-1$ if $r$ is a natural number or $\ell=\lfloor r\rfloor$ otherwise (note that $1 \leq r$ if $r$ is a natural number since we assumed that $0 \leq m<r)$. In both cases the integer $\ell$ is such that $m \leq \ell \leq k$. Let $\mathcal{A}_{\ell, d}=\left\{\alpha \in \mathbb{N}^{d}|| \alpha \mid:=\right.$ $\left.\alpha_{1}+\ldots+\alpha_{d} \leq \bar{\ell}\right\}$. Note that $\operatorname{card}\left(\mathcal{A}_{\ell, d}\right)=\operatorname{dim}\left(\mathbb{P}_{\ell, d}\right)=\left(\begin{array}{c}\ell+d \\ d\end{array}\right)=: N_{\ell, d}$. Since the mapping $\Phi_{\ell, d}: \mathbb{P}_{\ell, d} \rightarrow \mathbb{R}^{N_{\ell, d}}$ such that $\Phi_{\ell, d}(q)=\left(\int_{D_{K}} \partial^{\alpha} q \mathrm{~d} x\right)_{\alpha \in \mathcal{A}_{\ell, d}}$ is an isomorphism, there is a unique polynomial $\pi_{\ell}(v) \in \mathbb{P}_{\ell, d}$ such that $\Phi_{\ell, d}\left(\pi_{\ell}(v)\right)=\left(\int_{D_{K}} \partial^{\alpha} v \mathrm{~d} x\right)_{\alpha \in \mathcal{A}_{\ell, d}}$, i.e. $\int_{D_{K}} \partial^{\alpha}\left(v-\pi_{\ell}(v)\right) \mathrm{d} x=0$ for all $\alpha \in \mathcal{A}_{\ell, d}$ (this result is actually stated in (Morrey [19], Thm. 3.6.10).

Since by definition $\int_{D_{K}} \partial^{\alpha}\left(v-\pi_{\ell}(v)\right) \mathrm{d} x=0$ for all $|\alpha|=m \leq \ell$, we can apply Lemma 5.7 below, i.e. there is a uniform constant $c$ such that $\left|v-\pi_{\ell}(v)\right|_{W^{m, p}\left(D_{K}\right)} \leq c h_{K}\left|v-\pi_{\ell}(v)\right|_{W^{m+1, p}\left(D_{K}\right)}$. We can repeat the argument if $m+1 \leq \ell$ since in this case we also have $\int_{D_{K}} \partial^{\alpha}\left(v-\pi_{\ell}(v)\right) \mathrm{d} x=0$ for all $|\alpha|=m+1 \leq \ell$. Eventually, we obtain

$$
\left|v-\pi_{\ell}(v)\right|_{W^{m, p}\left(D_{K}\right)} \leq c h_{K}^{\ell-m}\left|v-\pi_{\ell}(v)\right|_{W^{\ell, p}\left(D_{K}\right)} .
$$

If $r$ is a natural number, then $\ell+1=r$, and we can apply the above argument one last time since $\int_{D_{K}} \partial^{\alpha}(v-$ $\left.\pi_{\ell}(v)\right) \mathrm{d} x=0$ for all $|\alpha|=\ell$, which then gives (5.7) because $\partial^{\alpha} \pi_{\ell}(v)=0$ for all $|\alpha|=\ell+1$. Otherwise, $\ell=\lfloor r\rfloor$ and we apply Lemma 7.1 to all the partial derivatives $\partial^{\alpha}\left(v-\pi_{\ell}(v)\right)$ with $|\alpha|=\ell, s=r-\lfloor r\rfloor \in(0,1)$ and $O:=D_{K}$; this is legitimate since all these partial derivatives have zero average over $D_{K}$. We infer that there is $c$ uniform with respect to $s, p, K$, and $v$ such that

$$
\left|v-\pi_{\ell}(v)\right|_{W^{m, p}\left(D_{K} ; \mathbb{R}^{q}\right)} \leq c h_{K}^{\lfloor r\rfloor-m} h_{D_{K}}^{r-\lfloor r\rfloor}\left(\frac{h_{D_{K}}^{d}}{\left|D_{K}\right|}\right)^{\frac{1}{p}}\left|v-\pi_{\ell}(v)\right|_{W^{r, p}\left(D_{K} ; \mathbb{R}^{q}\right)} .
$$

Note that $\left|v-\pi_{\ell}(v)\right|_{W^{r, p}\left(D_{K} ; \mathbb{R}^{q}\right)}=|v|_{W^{r, p}\left(D_{K} ; \mathbb{R}^{q}\right)}$ since $\partial^{\alpha} \pi_{\ell}(v)$ is a constant in $\mathbb{R}^{q}$ for all $|\alpha|=\ell$. We conclude that (5.7) holds owing to the shape-regularity of the mesh sequence.

Lemma 5.7 (Poincaré inequality in $D_{K}$ ). Let $\underline{w}_{D_{K}}$ be the average of $w$ over $D_{K}$. There exists a uniform constant $c$ such that

$$
\left\|v-\underline{v}_{D_{K}}\right\|_{L^{p}\left(D_{K}\right)} \leq c h_{K}|v|_{W^{1, p}\left(D_{K}\right)},
$$

for all $p \in[1, \infty]$, all $v \in W^{1, p}\left(D_{K}\right)$, and all $K \in \mathcal{T}_{h}$. 
Proof. Let $K \in \mathcal{T}_{h}$. Let $K_{l}, K_{r} \in \mathcal{T}_{K}$ sharing an interface $F=\partial K_{l} \cap \partial K_{r}$. We observe that

$$
\left|\underline{v}_{K_{l}}-\underline{v}_{K_{r}}\right|=|F|^{-\frac{1}{p}}\left\|_{\underline{v}_{K_{l}}}-v_{\mid K_{l}}+v_{\mid K_{r}}-\underline{v}_{K_{r}}\right\|_{L^{p}(F)},
$$

since $v_{\mid K_{l}}=v_{\mid K_{r}}$ on $F$. By using the triangle inequality, estimating the two norms in $L^{p}(F)$ with the trace inequality (7.2) (with $s=1$ ), and by applying the Poincaré inequality in $K_{l}$ and $K_{r}$ separately (both with constant $\pi^{-1}$ since the mesh cells are convex sets), we obtain $\left|\underline{v}_{K_{l}}-\underline{v}_{K_{r}}\right| \leq c\left(h_{K_{l}}^{1-\frac{1}{p}}|v|_{W^{1, p}\left(K_{l}\right)}+h_{K_{r}}^{1-\frac{1}{p}}|v|_{W^{1, p}\left(K_{r}\right)}\right)$. After invoking the shape regularity of the mesh sequence, we infer that

$$
|K|^{\frac{1}{p}}\left|\underline{v}_{K_{l}}-\underline{v}_{K_{r}}\right| \leq c h_{K}|v|_{W^{1, p}\left(K_{l} \cup K_{r}\right)} .
$$

Observing that $\underline{v}_{D_{K}}-\underline{v}_{K^{\prime}}=\sum_{K^{\prime \prime} \in \mathcal{T}_{K}} \frac{\left|K^{\prime \prime}\right|}{\left|D_{K}\right|}\left(\underline{v}_{K^{\prime \prime}}-\underline{v}_{K^{\prime}}\right)$ for any $K^{\prime} \in \mathcal{T}_{K}$, we infer that

$$
\left\|v-\underline{v}_{D_{K}}\right\|_{L^{p}\left(K^{\prime}\right)} \leq\left\|v-\underline{v}_{K^{\prime}}\right\|_{L^{p}\left(K^{\prime}\right)}+\sum_{K^{\prime \prime} \in \mathcal{T}_{K}} \frac{\left|K^{\prime \prime}\right|}{\left|D_{K}\right|}\left|K^{\prime}\right| \frac{1}{p}\left|\underline{v}_{K^{\prime \prime}}-\underline{v}_{K^{\prime}}\right| .
$$

For any $K^{\prime \prime} \in \mathcal{T}_{K}$, we can find a path of mesh cells in $\mathcal{T}_{K}$ linking $K^{\prime}$ to $K^{\prime \prime}$ so that any consecutive mesh cells in the path share a common face and this face is crossed only once. Using (5.9) together with the shape regularity of the mesh sequence, we infer that $\left\|v-\underline{v}_{K^{\prime}}\right\|_{L^{p}\left(K^{\prime}\right)} \leq c h_{K}|v|_{W^{1, p}\left(D_{K}\right)}$, and the conclusion follows by summing over $K^{\prime} \in \mathcal{T}_{h}$ and using the fact that $\operatorname{card}\left(\mathcal{T}_{K}\right)$ is uniformly bounded.

\section{QUASI-INTERPOLATION WITH BOUNDARY PRESCRIPTION}

Our goal in this section is to construct a variant of the quasi-interpolation operator $\mathcal{I}_{h}^{\text {av }}$ that prescribes homogeneous boundary values.

\subsection{Trace operator}

Let $F \in \mathcal{F}_{h}^{\partial}$ be a boundary face. We denote by $K_{F}$ the unique cell such that $F \subset \partial K_{F}$. We consider the global trace operator $\gamma: W^{1,1}\left(D ; \mathbb{R}^{q}\right) \rightarrow L^{1}\left(\partial D ; \mathbb{R}^{t}\right)$ such that

$$
\gamma(v)_{\mid F}=\gamma_{K_{F}, F}\left(v_{\mid K_{F}}\right), \quad \forall F \in \mathcal{F}_{h}^{\partial} .
$$

We define for all $p \in[1, \infty]$ the functional space

$$
W_{0, \gamma}^{1, p}\left(D ; \mathbb{R}^{q}\right):=\left\{v \in W^{1, p}\left(D ; \mathbb{R}^{q}\right) \mid \gamma(v)=0\right\} .
$$

We then set

$$
P_{0}\left(\mathcal{T}_{h}\right):=\left\{v_{h} \in P\left(\mathcal{T}_{h}\right) \mid \gamma\left(v_{h}\right)=0\right\} .
$$

The typical examples we have in mind are

$$
\begin{aligned}
& P_{0}^{\mathrm{g}}\left(\mathcal{T}_{h}\right):=\left\{v_{h} \in P^{\mathrm{g}}\left(\mathcal{T}_{h}\right) \mid v_{h \mid \partial D}=0\right\}, \\
& \boldsymbol{P}_{0}^{\mathrm{c}}\left(\mathcal{T}_{h}\right):=\left\{\boldsymbol{v}_{h} \in P^{\mathrm{c}}\left(\mathcal{T}_{h}\right) \mid \boldsymbol{v}_{h} \times \boldsymbol{n}_{\mid \partial D}=\mathbf{0}\right\}, \\
& \boldsymbol{P}_{0}^{\mathrm{d}}\left(\mathcal{T}_{h}\right):=\left\{\boldsymbol{v}_{h} \in P^{\mathrm{d}}\left(\mathcal{T}_{h}\right) \mid \boldsymbol{v}_{h} \cdot \boldsymbol{n}_{\mid \partial D}=0\right\} .
\end{aligned}
$$

Upon setting $V_{0}^{\mathrm{g}}=\left\{v \in V^{\mathrm{g}} \mid v_{\mid \partial D}=0\right\}, \boldsymbol{V}_{0}^{\mathrm{c}}=\left\{\boldsymbol{v} \in \boldsymbol{V}^{\mathrm{c}} \mid \boldsymbol{v} \times \boldsymbol{n}_{\mid \partial D}=\mathbf{0}\right\}$, and $\boldsymbol{V}_{0}^{\mathrm{d}}=\left\{\boldsymbol{v} \in \boldsymbol{V}^{\mathrm{d}} \mid \boldsymbol{v} \cdot \boldsymbol{n}_{\mid \partial D}=0\right\}$, we have $P_{0}^{\mathrm{g}}\left(\mathcal{T}_{h}\right)=P^{\mathrm{g}}\left(\mathcal{T}_{h}\right) \cap V_{0}^{\mathrm{g}}, \boldsymbol{P}_{0}^{\mathrm{c}}\left(\mathcal{T}_{h}\right)=\boldsymbol{P}^{\mathrm{c}}\left(\mathcal{T}_{h}\right) \cap \boldsymbol{V}_{0}^{\mathrm{c}}$, and $\boldsymbol{P}_{0}^{\mathrm{d}}\left(\mathcal{T}_{h}\right)=\boldsymbol{P}^{\mathrm{d}}\left(\mathcal{T}_{h}\right) \cap \boldsymbol{V}_{0}^{\mathrm{d}}$, i.e. the finite element spaces $P_{0}^{\mathrm{g}}\left(\mathcal{T}_{h}\right), \boldsymbol{P}_{0}^{\mathrm{c}}\left(\mathcal{T}_{h}\right), \boldsymbol{P}_{0}^{\mathrm{d}}\left(\mathcal{T}_{h}\right)$ are conforming in $V_{0}^{\mathrm{g}}, \boldsymbol{V}_{0}^{\mathrm{c}}, \boldsymbol{V}_{0}^{\mathrm{d}}$, respectively.

In the rest of the paper, we slightly abuse the terminology by calling global degrees of freedom the elements of $\mathcal{A}_{h}$. We say that a global degree of freedom $a \in \mathcal{A}_{h}$ is an internal degree of freedom if $\gamma\left(\varphi_{a}\right)=0$. The collection 
of all the internal degrees of freedom is denoted $\mathcal{A}_{h}^{\circ}$; the degrees of freedom in $\mathcal{A}_{h}^{\partial}=\mathcal{A}_{h} \backslash \mathcal{A}_{h}^{\circ}$ are called boundary degrees of freedom. Let $a \in \mathcal{A}_{h}^{\partial}$, then there is a face $F \in \mathcal{F}_{h}^{\partial}$ such that $\gamma\left(\varphi_{a}\right)_{\mid F} \neq 0$. Let $K_{F}$ be the unique cell such that $F \subset \partial K_{F}$, then $\operatorname{supp}\left(\varphi_{a}\right) \cap K_{F} \neq \emptyset$. This means that there is a unique $i_{F} \in \mathcal{N}$ such that $\varphi_{a \mid K_{F}}=\theta_{K_{F}, i}$. For all $a \in \mathcal{A}_{h}^{\partial}$, we define $\mathcal{F}_{a}^{\partial}$ to be the collection of all the boundary faces $F \in \mathcal{F}_{h}^{\partial}$ such that there is $\left(K_{F}, i_{F}\right) \in \mathcal{C}_{a}$ and $F \subset \partial K_{F}$; we set $\mathcal{F}_{a}^{\partial}=\emptyset$ if $a \in \mathcal{A}_{h}^{\circ}$. We abuse the notation by setting

$$
\llbracket v \rrbracket_{F}^{\gamma}(\boldsymbol{x})=\gamma_{K_{F}, F}\left(v_{\mid K_{F}}\right)(\boldsymbol{x}), \quad \text { and } \llbracket v \rrbracket_{F}(\boldsymbol{x})=v_{\mid F}(\boldsymbol{x}), \quad \text { a.e. } \boldsymbol{x} \in F, \forall F \in \mathcal{F}_{h}^{\partial},
$$

and assume that $\left|\llbracket v \rrbracket_{F}^{\gamma}(\boldsymbol{x})\right| \leq\left|\llbracket v \rrbracket_{F}(\boldsymbol{x})\right|$, a.e. $\boldsymbol{x} \in F$, for all $F \in \mathcal{F}_{h}^{\partial}$. In coherence with the assumption (4.2), we assume that there is a uniform constant $c$ such that the following holds for all the boundary degrees of freedom $a \in \mathcal{A}_{h}^{\partial}$, all $F \in \mathcal{F}_{a}^{\partial}$, all $i_{F} \in \mathcal{N}$ such that $\left(K_{F}, i_{F}\right) \in \mathcal{C}_{a}$, and all $v \in P^{\mathrm{b}}\left(\mathcal{T}_{h}\right)$ :

$$
\left|\sigma_{K_{F}, i_{F}}(v)\right| \leq c\left\|\mathbb{A}_{K_{F}}\right\|_{\ell^{2}}\left\|\gamma_{K_{F}, F}\left(v_{\mid K_{F}}\right)\right\|_{L^{\infty}\left(F ; \mathbb{R}^{t}\right)},
$$

Note that this assumption is satisfied by all the finite elements considered in Section 2.4.

\subsection{Averaging and quasi-interpolation operators revisited}

We are going to modify the averaging operator $\mathcal{J}_{h}^{\text {av }}$ to prescribe homogeneous boundary conditions. We define $\mathcal{J}_{h 0}^{\text {av }}: P^{\mathrm{b}}\left(\mathcal{T}_{h}\right) \rightarrow P_{0}\left(\mathcal{T}_{h}\right)$ by setting for all $v \in P^{\mathrm{b}}\left(\mathcal{T}_{h}\right)$,

$$
\mathcal{J}_{h 0}^{\mathrm{av}}(v)=\sum_{a \in \mathcal{A}_{h}^{\circ}}\left(\frac{1}{\operatorname{card}\left(\mathcal{C}_{a}\right)} \sum_{(K, i) \in \mathcal{C}_{a}} \sigma_{K, i}\left(v_{\mid K}\right)\right) \varphi_{a} .
$$

Lemma 6.1 ( $L^{p}$-stability). There exists a uniform constant $c$ such that

$$
\left\|\mathcal{J}_{h 0}^{\mathrm{av}}(v)\right\|_{L^{p}\left(K ; \mathbb{R}^{q}\right)} \leq c\|v\|_{L^{p}\left(D_{K} ; \mathbb{R}^{q}\right)},
$$

for all $p \in[1, \infty]$, all $v \in P^{\mathrm{b}}\left(\mathcal{T}_{h}\right)$, and all $K \in \mathcal{T}_{h}$.

Proof. Proceed as in the proof of Lemma 4.2.

Lemma 6.2 (Approximation by averaging). There exists a uniform constant $c$ such that the following holds:

$$
\left|w-\mathcal{J}_{h 0}^{\mathrm{av}}(w)\right|_{W^{m, p}\left(K ; \mathbb{R}^{q}\right)} \leq c h_{K}^{d\left(\frac{1}{p}-\frac{1}{r}\right)+\frac{1}{r}-m} \sum_{F \in \mathcal{F}_{K}^{\circ} \cup \mathcal{F}_{K}^{\partial}}\left\|\llbracket w \rrbracket_{F}^{\gamma}\right\|_{L^{r}\left(F ; \mathbb{R}^{t}\right)},
$$

for all $m \in\{0: k+1\}$, all $p, r \in[1, \infty]$, all $w \in P^{\mathrm{b}}\left(\mathcal{T}_{h}\right)$, and all $K \in \mathcal{T}_{h}$, with $\mathcal{F}_{K}^{\partial}=\cup_{i \in \mathcal{N}} \mathcal{F}_{\mathrm{a}(K, i)}^{\partial}$ and $\mathcal{F}_{K}^{\circ}$ defined in Lemma 4.3.

Proof. This is a straightforward adaptation of the proof of Lemma 4.3. Letting $e=v-\mathcal{J}_{h 0}^{\text {av }}(v)$, we observe that $\sigma_{K, i}\left(e_{\mid K}\right)$ is still given by (4.8) if a $(K, i) \in \mathcal{A}_{h}^{\circ}$, while $\sigma_{K, i}\left(e_{\mid K}\right)=\sigma_{K, i}(w)$ if a $(K, i) \in \mathcal{A}_{h}^{\partial}$, and this term is bounded using (6.6).

A global quasi-interpolation operator $\mathcal{I}_{h 0}^{\text {av }}: L^{1}\left(D ; \mathbb{R}^{q}\right) \rightarrow P_{0}\left(\mathcal{T}_{h}\right)$ is then defined by setting

$$
\mathcal{I}_{h 0}^{\text {av }}=\mathcal{J}_{h 0}^{\text {av }} \circ \mathcal{I}_{h}^{\sharp} \text {. }
$$

Note that $P_{0}\left(\mathcal{T}_{h}\right)$ is point-wise invariant under $\mathcal{I}_{h 0}^{\text {av }}$ since $(6.9)$ implies that $P_{0}\left(\mathcal{T}_{h}\right)$ is point-wise invariant under $\mathcal{J}_{h 0}^{\text {av }}$. Hence, $\mathcal{I}_{h 0}^{\text {av }}$ is a projection, i.e. $\left(\mathcal{I}_{h 0}^{\text {av }}\right)^{2}=\mathcal{I}_{h 0}^{\text {av }}$.

Lemma 6.3 ( $L^{p}$-stability of $\left.\mathcal{I}_{h 0}^{\text {av }}\right)$. There is a uniform constant c such that

$$
\left\|\mathcal{I}_{h 0}^{\mathrm{av}}(v)\right\|_{L^{p}\left(K ; \mathbb{R}^{q}\right)} \leq c\|v\|_{L^{p}\left(D_{K} ; \mathbb{R}^{q}\right)}
$$

for all $p \in[1, \infty]$, all $v \in L^{p}\left(D ; \mathbb{R}^{q}\right)$, and all $K \in \mathcal{T}_{h}$.

Proof. Proceed as for the proof of Lemma 5.1. 


\subsection{Error estimates}

The purpose of this section is to establish error estimates for the quasi-interpolation operator $\mathcal{I}_{h 0}^{\text {av }}$ in the $W^{r, p}$-norm (either integer or fractional). Let $r \in[0, k+1]$ and $p \in[1, \infty)$ if $r \notin \mathbb{N}$ or $p \in[1, \infty]$ if $r \in \mathbb{N}$. If $r>\frac{1}{p}$, then functions in $W^{r, p}\left(D ; \mathbb{R}^{q}\right)$ have traces on $\partial D$, and therefore it makes sense to define

$$
W_{0, \gamma}^{r, p}\left(D ; \mathbb{R}^{q}\right):=\left\{v \in W^{r, p}\left(D ; \mathbb{R}^{q}\right) \mid \gamma(v)=0\right\} .
$$

We are going to use the following notation

$$
\begin{aligned}
& \mathcal{T}_{h}^{\circ}:=\left\{K \in \mathcal{T}_{h} \mid \forall i \in \mathcal{N}, \mathrm{a}(K, i) \in \mathcal{A}_{h}^{\circ}\right\}, \\
& \mathcal{T}_{h}^{\partial}:=\mathcal{T}_{h} \backslash \mathcal{T}_{h}^{\circ}=\left\{K \in \mathcal{T}_{h} \mid \exists i \in \mathcal{N}, \mathrm{a}(K, i) \in \mathcal{A}_{h}^{\partial}\right\}, \\
& D^{\partial}:=\operatorname{int}\left(\cup_{K \in \mathcal{T}_{h}^{\partial}} K\right) .
\end{aligned}
$$

$\mathcal{T}_{h}^{\circ}$ is the set of the cells whose degrees of freedom are all internal. $\mathcal{T}_{h}^{\partial}$ is the set of the cells that have at least one boundary degree of freedom. $D^{\partial}$ is the collection of the points in $D$ that belong to at least one cell in $\mathcal{T}_{h}^{\partial}$.

Theorem 6.4 (Approximation). There exists a uniform constant $c$ such that the following estimate holds for all $r \in[0, k+1]$, all $m \in\{0:\lfloor r\rfloor\}$, all $p \in[1, \infty)$ if $r \notin \mathbb{N}$ or all $p \in[1, \infty]$ if $r \in \mathbb{N}$, all $v \in W^{r, p}\left(D_{K} ; \mathbb{R}^{q}\right)$, and all $K \in \mathcal{T}_{h}^{\circ}$ :

$$
\left|v-\mathcal{I}_{h 0}^{\mathrm{av}}(v)\right|_{W^{m, p}\left(K ; \mathbb{R}^{q}\right)} \leq c h_{K}^{r-m}|v|_{W^{r, p}\left(D_{K} ; \mathbb{R}^{q}\right)} .
$$

Moreover, (6.14) also holds if $r p>1$ with $c$ depending on $|r p-1|$ for all $v \in W_{0, \gamma}^{r, p}\left(D ; \mathbb{R}^{q}\right)$ and all $K \in \mathcal{T}_{h}^{\partial}$. Finally, if $r p<1$ (i.e. $r \in(0,1)$ and $m=0$ ), we have

$$
\left\|v-\mathcal{I}_{h 0}^{\mathrm{av}}(v)\right\|_{L^{p}\left(D^{\partial ;} ; \mathbb{R}^{q}\right)} \leq c h^{r}\|v\|_{W^{r, p}\left(D ; \mathbb{R}^{q}\right)}, \quad \forall v \in W^{r, p}\left(D ; \mathbb{R}^{q}\right) .
$$

Proof. Let $K$ be a cell in $\mathcal{T}_{h}$. If $K \in \mathcal{T}_{h}^{\circ}$, then $\mathcal{I}_{h 0}^{\text {av }}(v)_{\mid K}=\mathcal{I}_{h}^{\text {av }}(v)_{\mid K}$; this proves (6.14) in this case. Let us now consider $K \in \mathcal{T}_{h}^{\partial}$. The triangle inequality implies that

$$
\left|\mathcal{I}_{h 0}^{\text {av }}(v)-v\right|_{W^{m, p}\left(K ; \mathbb{R}^{q}\right)} \leq\left|\mathcal{I}_{h}^{\text {av }}(v)-v\right|_{W^{m, p}\left(K ; \mathbb{R}^{q}\right)}+\left|\mathcal{I}_{h 0}^{\text {av }}(v)-\mathcal{I}_{h}^{\text {av }}(v)\right|_{W^{m, p}\left(K ; \mathbb{R}^{q}\right)} .
$$

Since we have already established that $\left|\mathcal{I}_{h}^{\text {av }}(v)-v\right|_{W^{m, p}\left(K ; \mathbb{R}^{q}\right)} \leq c h_{K}^{r-m}|v|_{W^{r, p}\left(D_{K} ; \mathbb{R}^{q}\right)}$ in Theorem 5.2, we just need to estimate $\left|\mathcal{I}_{h 0}^{\text {av }}(v)-\mathcal{I}_{h}^{\text {av }}(v)\right|_{W^{m, p}\left(K ; \mathbb{R}^{q}\right)}$. Let us define the set of the boundary degrees of freedom with nonempty support on $K, \mathcal{A}_{K}^{\partial}:=\left\{a \in \mathcal{A}_{h}^{\partial} \mid \exists i \in \mathcal{N}, \mathrm{a}(K, i) \in \mathcal{C}_{a}\right\}$. Then

$$
\left(\mathcal{I}_{h 0}^{\mathrm{av}}(v)-\mathcal{I}_{h}^{\mathrm{av}}(v)\right)_{\mid K}=-\sum_{a \in \mathcal{A}_{K}^{\partial}}\left(\frac{1}{\operatorname{card}\left(\mathcal{C}_{a}\right)} \sum_{\left(K^{\prime}, i^{\prime}\right) \in \mathcal{C}_{a}} \sigma_{\left(K^{\prime}, i^{\prime}\right)}\left(\mathcal{I}_{K^{\prime}}^{\sharp}\left(\left.v\right|_{K^{\prime}}\right)\right)\right) \theta_{K, i} .
$$

For any $a \in \mathcal{A}_{K}^{\partial}$ and any $\left(K^{\prime}, i^{\prime}\right) \in \mathcal{C}_{a}$, there is a face $F \in \mathcal{F}_{a}^{\partial}$ and a pair $\left(K_{F}, i_{F}\right) \in \mathcal{C}_{a}$ such that there is a path of mesh cells in $\mathcal{T}_{K}$ linking $K^{\prime}$ to $K_{F}$ so that any two consecutive mesh cells in the path share a common face in $\mathcal{F}_{a}^{\circ}$, and each face is crossed only once. This observation implies that

$$
\left|\sigma_{\left(K^{\prime}, i^{\prime}\right)}(g)\right| \leq \sum_{F \in \mathcal{F}_{a}^{\circ}}\left|\sigma_{\mathrm{a}\left(K_{l}, i_{l}\right)}\left(g_{\mid K_{l}}\right)-\sigma_{\mathrm{a}\left(K_{r}, i_{r}\right)}\left(g_{\mid K_{r}}\right)\right|+\sum_{F \in \mathcal{F}_{a}^{\partial}}\left|\sigma_{\mathrm{a}\left(K_{F}, i_{F}\right)}\left(g_{\mid K_{F}}\right)\right|
$$

for all $g \in P^{\mathrm{b}}\left(\mathcal{T}_{h}\right)$. By proceeding as in the proof of Lemma 4.3, we infer that

$$
\left|\mathcal{I}_{h 0}^{\mathrm{av}}(v)-\mathcal{I}_{h}^{\mathrm{av}}(v)\right|_{W^{m, p}\left(K ; \mathbb{R}^{q}\right)} \leq c h_{K}^{-m+\frac{1}{p}} \sum_{a \in \mathcal{A}_{K}^{\partial}}\left[\sum_{F \in \mathcal{F}_{a}^{\circ}}\left\|\llbracket \mathcal{I}^{\sharp}(v) \rrbracket_{F}^{\gamma}\right\|_{L^{p}\left(F ; \mathbb{R}^{t}\right)}+\sum_{F \in \mathcal{F}_{a}^{\partial}}\left\|\gamma\left(\mathcal{I}^{\sharp}(v)\right)_{\mid F}\right\|_{L^{p}\left(F ; \mathbb{R}^{t}\right)}\right] .
$$


Case 1, $\boldsymbol{r p}>1$ : Let us assume that $v \in W_{0, \gamma}^{r, p}\left(D ; \mathbb{R}^{q}\right)$. The boundary condition $\gamma(v)_{F}=0$, for all $F \in \mathcal{F}_{h}^{\partial}$, and the continuity condition $\llbracket v \rrbracket_{F}^{\gamma}=0$, for all $F \in \mathcal{F}_{h}^{\circ}$, imply that

$$
\left|\mathcal{I}_{h 0}^{\mathrm{av}}(v)-\mathcal{I}_{h}^{\mathrm{av}}(v)\right|_{W^{m, p}\left(K ; \mathbb{R}^{q}\right)} \leq c h_{K}^{-m+\frac{1}{p}} \sum_{F \in \mathcal{F}_{K}^{\circ} \cup \mathcal{F}_{K}^{\partial}}\left\|\mathcal{I}_{K}^{\sharp}(v)-v\right\|_{L^{p}\left(F ; \mathbb{R}^{t}\right)} .
$$

The conclusion follows by invoking the trace inequality from Lemma 7.2, either with $s=1$ if $r \in \mathbb{N}$ or with $s=r-\lfloor r\rfloor$ otherwise, and the approximation properties of $\mathcal{I}_{K}^{\sharp}$ stated either in Theorem 3.3 or in Remark 5.5.

Case 2, $\boldsymbol{r p}<\mathbf{1}$ : Assume now that $r p<1$. Norm equivalence implies that

$$
\left.\left\|\mathcal{I}_{h 0}^{\mathrm{av}}(v)-\mathcal{I}_{h}^{\mathrm{av}}(v)\right\|_{L^{p}\left(D^{\partial} ; \mathbb{R}^{q}\right)}^{p} \leq c \| \mathcal{I}_{h}^{\sharp}(v)\right) \|_{L^{p}\left(D^{\partial} ; \mathbb{R}^{q}\right)}^{p} .
$$

Let $\rho$ be the distance to $\partial D$; then there is $c$ uniform with respect to the mesh sequence such that $\|\rho\|_{L^{\infty}\left(D^{\partial}\right)} \leq c h$ and

$$
\begin{aligned}
\left\|\mathcal{I}_{h 0}^{\mathrm{av}}(v)-\mathcal{I}_{h}^{\mathrm{av}}(v)\right\|_{L^{p}\left(D^{\partial} ; \mathbb{R}^{q}\right)} & \leq c\left(\left\|\mathcal{I}_{h}^{\sharp}(v)-v\right\|_{L^{p}\left(D^{\partial} ; \mathbb{R}^{q}\right)}+\|v\|_{L^{p}\left(D^{\partial} ; \mathbb{R}^{q}\right)}\right) \\
& \leq c\left(h^{r}|v|_{W^{r, p}\left(D ; \mathbb{R}^{q}\right)}+\left\|\rho^{r} \rho^{-r} v\right\|_{L^{p}\left(D^{\partial} ; \mathbb{R}^{q}\right)}\right) \\
& \leq c\left(h^{r}|v|_{W^{r, p}\left(D ; \mathbb{R}^{q}\right)}+\|\rho\|_{L^{\infty}\left(D^{\partial}\right)}^{r}\left\|\rho^{-r} v\right\|_{L^{p}\left(D^{\partial} ; \mathbb{R}^{q}\right)}\right) .
\end{aligned}
$$

Since $r p<1$, we infer that (see Grisvard [16], Cor. 1.4.4.5)

$$
\left\|\rho^{-r} v\right\|_{L^{p}\left(D^{\partial} ; \mathbb{R}^{q}\right)} \leq\left\|\rho^{-r} v\right\|_{L^{p}\left(D ; \mathbb{R}^{q}\right)} \leq c\|v\|_{W^{r, p}\left(D ; \mathbb{R}^{q}\right)} .
$$

In conclusion, $\left\|\mathcal{I}_{h 0}^{\text {av }}(v)-\mathcal{I}_{h}^{\text {av }}(v)\right\|_{L^{p}\left(D^{\partial} ; \mathbb{R}^{q}\right)} \leq c h^{r}\|v\|_{W^{r, p}\left(D ; \mathbb{R}^{q}\right)}$. Note that the constant $c$ scales like $\ell_{D}^{-r}$ where $\ell_{D}$ is the diameter of $D$.

Corollary 6.5 (Global best approximation in $L^{p}$ ). There exists a uniform constant $c$, additionally depending on $|r p-1|$, such that

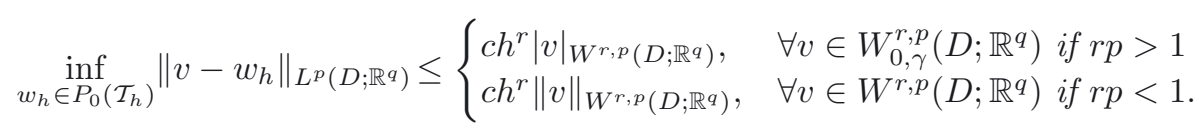

Remark 6.6 (Theorem 6.4). For $r p>1$, a similar estimate has been obtained in the scalar-valued case for the Scott-Zhang interpolation operator by Ciarlet [7]. Furthermore, the estimate for $r p<1$ in Theorem 6.4 essentially says that the difference $v-\mathcal{I}_{h 0}^{\text {av }}(v)$ does not blow up too fast close to the boundary. A better result is not expected since $\mathcal{I}_{h 0}^{\text {av }}(v)$ is forced to be zero at $\partial D$ whereas $v$ can blow up like $\rho^{-s} w$ where $w$ is a function in $L^{p}\left(D ; \mathbb{R}^{q}\right)$.

Remark 6.7 $(r p=1)$. Let $r \in(0,1)$. Using the notation from the real interpolation theory, it is known that $W^{r, p}(D)=\left[L^{p}(D), W^{1, p}(D)\right]_{r, p}$ since $D$ is Lipschitz, (see Tartar [24], Lem. 36.1). Let us define

$$
W_{00, \gamma}^{r, p}\left(D ; \mathbb{R}^{q}\right):=\left[L^{p}\left(D ; \mathbb{R}^{q}\right), W_{0, \gamma}^{1, p}\left(D ; \mathbb{R}^{q}\right)\right]_{r, p} .
$$

Then, using Theorem 6.4 with $l \in\{0,1\}$ and $m=0$, the real interpolation theory implies that

$$
\left\|v-\mathcal{I}_{h 0}^{\mathrm{av}}(v)\right\|_{L^{p}\left(D ; \mathbb{R}^{q}\right)} \leq c h^{r}\|v\|_{W_{0, \gamma}^{r, p}\left(D ; \mathbb{R}^{q}\right)},
$$

for all $p \in[1, \infty)$ and all $v \in W_{00, \gamma}^{r, p}\left(D ; \mathbb{R}^{q}\right)$. This estimate is not fully satisfactory for two reasons. First it is not local. Second it is not really clear what $W_{00, \gamma}^{\frac{1}{p}, p}\left(D ; \mathbb{R}^{q}\right)$ is. For instance, let us define

$$
\begin{aligned}
& W_{0}^{1, p}(D):=\left\{v \in W^{1, p}(D) \mid v_{\mid \partial D}=0\right\}, \\
& \boldsymbol{W}_{T}^{1, p}(D):=\left\{\boldsymbol{v} \in \boldsymbol{W}^{1, p}(D) \mid \boldsymbol{v} \times \boldsymbol{n}_{\mid \partial D}=\mathbf{0}\right\}, \\
& \boldsymbol{W}_{N}^{1, p}(D):=\left\{\boldsymbol{v} \in \boldsymbol{W}^{1, p}(D) \mid \boldsymbol{v} \cdot \boldsymbol{n}_{\mid \partial D}=0\right\} .
\end{aligned}
$$


One then realizes that characterizing $\left[\boldsymbol{L}^{p}(D), \boldsymbol{W}_{T}^{1, p}(D)\right]_{\frac{1}{p}, p}$ and $\left[\boldsymbol{L}^{p}(D), \boldsymbol{W}_{N}^{1, p}(D)\right]_{\frac{1}{p}, p}$ in terms of Sobolev regularity is (possible but) not straightforward, and to the best of our knowledge, a full characterization of these spaces is not yet available.

\section{TeChnical Results in fRaCtional Sobolev SPACES}

This section contains two technical results in fractional Sobolev spaces: a Poincaré inequality and a trace inequality.

Lemma 7.1 (Poincaré inequality). Let $O$ be an open set in $\mathbb{R}^{d}$ and let $\underline{v}_{O}$ be the average of $v$ over $O$, for any $v \in L^{1}(O)$. Let $h_{O}:=\operatorname{diam}(O)$. Then, for all $v \in W^{s, p}(O)$ with $s \in(0,1)$ and $p \in[1, \infty)$, the following holds:

$$
\left\|v-\underline{v}_{O}\right\|_{L^{p}(O)} \leq h_{O}^{s}\left(\frac{h_{O}^{d}}{|O|}\right)^{\frac{1}{p}}|v|_{W^{s, p}(O)} .
$$

Proof. This result is essentially Proposition 6.1 from Dupont and Scott [12], see also Heuer [17]. We nevertheless give a proof since the computation gives us the constant in the right-hand side of (7.1), and this in turn allows us to apply the result locally on shape-regular meshes. Using the definitions, we have

$$
\begin{aligned}
\int_{O}\left|v(\boldsymbol{x})-\underline{v}_{O}\right|^{p} \mathrm{~d} x & =\int_{O}|O|^{-p}\left|\int_{O}(v(\boldsymbol{x})-v(\boldsymbol{y})) \mathrm{d} y\right|^{p} \mathrm{~d} x \\
& \leq \int_{O}|O|^{-p}\left(\int_{O} \frac{|v(\boldsymbol{x})-v(\boldsymbol{y})|}{\|\boldsymbol{x}-\boldsymbol{y}\|_{\ell^{2}}^{s+\frac{d}{p}}}\|\boldsymbol{x}-\boldsymbol{y}\|_{\ell^{2}}^{s+\frac{d}{p}} \mathrm{~d} y\right)^{p} \mathrm{~d} x \\
& \leq \int_{O}|O|^{-p} \int_{O} \frac{|v(\boldsymbol{x})-v(\boldsymbol{y})|^{p}}{\|\boldsymbol{x}-\boldsymbol{y}\|_{\ell^{2}}^{s p+d}} \mathrm{~d} y\left(\int_{O}\|\boldsymbol{x}-\boldsymbol{y}\|_{\ell^{2}}^{\left(s+\frac{d}{p}\right) p^{\prime}} \mathrm{d} y\right)^{\frac{p}{p^{\prime}}} \mathrm{d} x
\end{aligned}
$$

where $p^{\prime}:=\frac{p}{p-1}$. Then using that $\|\boldsymbol{x}-\boldsymbol{y}\|_{\ell^{2}} \leq h_{O}$ for all $\boldsymbol{x}, \boldsymbol{y} \in O$, we infer that

$$
\begin{aligned}
\left\|v-\underline{v}_{O}\right\|_{L^{p}(O)}^{p} & \leq \int_{O}|O|^{-p} \int_{O} \frac{|v(\boldsymbol{x})-v(\boldsymbol{y})|^{p}}{\|\boldsymbol{x}-\boldsymbol{y}\|_{\ell^{2}}^{s p+d}} \mathrm{~d} y \mathrm{~d} x\left(\max _{\boldsymbol{x} \in O} \int_{O}\|\boldsymbol{x}-\boldsymbol{y}\|_{\ell^{2}}^{\left(s+\frac{d}{p}\right) p^{\prime}} \mathrm{d} y\right)^{\frac{p}{p^{\prime}}} \\
& \leq|v|_{W^{s, p}(O)}^{p}|O|^{-p}\left(\int_{O} h_{O}^{\left(s+\frac{d}{p}\right) p^{\prime}} \mathrm{d} y\right)^{\frac{p}{p^{\prime}}} \\
& \leq|v|_{W^{s, p}(O)}^{p}|O|^{-p}|O|^{\frac{p}{p^{\prime}}} h_{O}^{s p+d} \leq|v|_{W^{s, p}(O)}^{p} h_{O}^{s p+d}|O|^{-1} .
\end{aligned}
$$

Hence $\left\|v-\underline{v}_{O}\right\|_{L^{p}(O)} \leq h_{O}^{s}\left(\frac{h_{O}^{d}}{|O|}\right)^{\frac{1}{p}}|v|_{W^{s, p}(O)}$.

Lemma 7.2 (Trace inequality). Assume $s \in(0,1)$ and $s p>1$ with $p \in[1, \infty)$ or $s=1$ with $p \in[1, \infty]$. There exists $c$, uniform with respect to the mesh sequence but depending on $|s p-1|$ if $s \in(0,1)$, such that the following holds for all $v \in W^{s, p}(K)$ and all $K \in \mathcal{T}_{h}$ :

$$
\|v\|_{L^{p}(F)} \leq c\left(h_{K}^{-\frac{1}{p}}\|v\|_{L^{p}(K)}+h_{K}^{s-\frac{1}{p}}|v|_{W^{s, p}(K)}\right) .
$$

Proof. Let $v \in W^{s, p}(D)$. Let $K \in \mathcal{T}_{h}$ be a cell and $F$ be a face of $K$. Since the map $\boldsymbol{T}_{K}$ is affine, using a trace inequality in $W^{s, p}(\widehat{K})$ (recall that $s \in(0,1)$ and $s p>1$ or $s=1$ and $p \geq 1$ ), we infer that

$$
\|v\|_{L^{p}(F)}=\frac{|F|^{\frac{1}{p}}}{|\widehat{F}|^{\frac{1}{p}}}\left\|\psi_{K}^{\mathrm{g}}(v)\right\|_{L^{p}(\widehat{F})} \leq c_{s, p}|F|^{\frac{1}{p}}\left(\left\|\psi_{K}^{\mathrm{g}}(v)\right\|_{L^{p}(\widehat{K})}+\left|\psi_{K}^{\mathrm{g}}(v)\right|_{W^{s, p}(\widehat{K})}\right),
$$


where $c_{s, p}$ depends on $|s p-1|$ if $s \in(0,1)$. Upon changing variables, this inequality is re-written

$$
\|v\|_{L^{p}(F)} \leq c_{s, p}|F|^{\frac{1}{p}}|K|^{-\frac{1}{p}}\left(\|v\|_{L^{p}(K)}+\left\|\mathbb{d}_{K}\right\|_{\ell^{2}}^{-s}|v|_{W^{s, p}(K)}\right) .
$$

The conclusion follows from the shape-regularity of the mesh sequence, see (2.2).

\section{REFERENCES}

[1] Y. Achdou, C. Bernardi and F. Coquel, A priori and a posteriori analysis of finite volume discretizations of Darcy's equations. Numer. Math. 96 (2003) 17-42.

[2] D.N. Arnold, R.S. Falk and R. Winther, Finite element exterior calculus, homological techniques, and applications. Acta Numer. 15 (2006) 1-155.

[3] C. Bernardi and V. Girault, A local regularization operator for triangular and quadrilateral finite elements. SIAM J. Numer. Anal. 35 (1998) 1893-1916.

[4] J.H. Bramble and S.R. Hilbert, Estimation of linear functionals on Sobolev spaces with application to Fourier transforms and spline interpolation. SIAM J. Numer. Anal. 7 (1970) 112-124.

[5] E. Burman and A. Ern, Continuous interior penalty $h p$-finite element methods for advection and advection-diffusion equations. Math. Comput. 76 (2007) 1119-1140.

[6] M. Campos Pinto and E. Sonnendrücker, Gauss-compatible Galerkin schemes for time-dependent Maxwell equations. Math. Comput. 302 (2016) 2651-2685.

[7] P. Ciarlet, Jr, Analysis of the Scott-Zhang interpolation in the fractional order Sobolev spaces. J. Numer. Math. 21 (2013) $173-180$.

[8] P.G. Ciarlet, The finite element method for elliptic problems, Vol. 40 of Classics in Applied Mathematics. Society for Industrial and Applied Mathematics (SIAM). Reprint of the 1978 original [North-Holland, Amsterdam; MR0520174 (58 \#25001)]. Philadelphia, PA (2002).

[9] P. Clément, Approximation by finite element functions using local regularization. RAIRO: Anal. Numer. 9 (1975) 77-84.

[10] B. Cockburn, G. Kanschat and D. Schötzau, A note on discontinuous Galerkin divergence-free solutions of the Navier-Stokes equations. J. Sci. Comput. 31 (2007) 61-73.

[11] D. Di Pietro and A. Ern, Mathematical aspects of discontinuous Galerkin methods. Vol. 69 of Mathématiques 83 Applications (Berlin) [Mathematics \& Applications]. Springer, Heidelberg (2012).

[12] T. Dupont and R. Scott, Polynomial approximation of functions in Sobolev spaces. Math. Comput. 34 (1980) $441-463$.

[13] A. Ern and J.-L. Guermond, Theory and practice of finite elements. Vol. 159 of Appl. Math. Sci. Springer-Verlag, New York (2004).

[14] A. Ern, A.F. Stephansen and M. Vohralík, Guaranteed and robust discontinuous Galerkin a posteriori error estimates for convection-diffusion-reaction problems. J. Comput. Appl. Math. 234 (2010) 114-130.

[15] V. Girault and J.-L. Lions, Two-grid finite-element schemes for the transient Navier-Stokes problem. ESAIM: M2AN 35 (2001) 945-980.

[16] P. Grisvard, Elliptic problems in nonsmooth domains. Vol. 24 of Monographs and Studies in Mathematics. Pitman (Advanced Publishing Program), Boston, MA (1985).

[17] N. Heuer, On the equivalence of fractional-order Sobolev semi-norms. J. Math. Anal. Appl. 417 (2014) $505-518$.

[18] O.A. Karakashian and F. Pascal, A posteriori error estimates for a discontinuous Galerkin approximation of second-order elliptic problems. SIAM J. Numer. Anal. 41 (2003) 2374-2399.

[19] C.B. Morrey, Jr, Mettre en romain : Multiple integrals in the calculus of variations. Die Grundlehren der Mathematischen Wissenschaften, Band 130. Springer-Verlag New York, Inc., New York (1966).

[20] P. Oswald, On a BPX-preconditioner for P1 elements. Computing 51 (1993) 125-133.

[21] A.C. Ponce and J. Van Schaftingen, The continuity of functions with $N$-th derivative measure. Houston J. Math. 33 (2007) 927-939.

[22] J. Schöberl and C. Lehrenfeld, Domain decomposition preconditioning for high order hybrid discontinuous Galerkin methods on tetrahedral meshes. In Advanced finite element methods and applications. Vol. 66 of Lect. Notes Appl. Comput. Mech. Springer, Heidelberg (2013) 27-56.

[23] R.L. Scott and S. Zhang, Finite element interpolation of nonsmooth functions satisfying boundary conditions. Math. Comput. 54 (1990) 483-493.

[24] L. Tartar, An introduction to Sobolev spaces and interpolation spaces. Vol. 3 of Lecture Notes of the Unione Matematica Italiana. Springer, Berlin; UMI, Bologna (2007). 